\title{
Katharina König \\ Prosodie und epistemic stance: Konstruktionen mit finalem oder
}

\section{Einleitung}

Die Untersuchung von Konstruktionen in ihrem handlungs- und sequenzgebundenen Gebrauch bildet den Ausgangspunkt der Interaktionalen Konstruktionsgrammatik (Günthner 2009; Imo 2016; Linell 2009; siehe auch Deppermann 2011): Form-Bedeutungs-Paare sind auf spezifische Verwendungskontexte zugeschnitten; sie haben sich im Gebrauch sedimentiert und werden stets in diesem re-aktualisiert. Insbesondere am Rand von Äußerungen oder Äußerungseinheiten haben sich zur Bearbeitung verschiedener diskurspragmatischer Aufgaben rekurrente Strukturen herausgebildet: So erfolgt die Verknüpfung sprachlicher Handlungen etwa über initial positionierte, syntaktisch desintegrierte Diskursmarker, die sich u. a. aus Subjunktionen, Imperativen oder Matrixsatzkonstruktionen entwickelt haben (Günthner 2017; Imo 2012). Über äußerungsinitiale Partikeln können zudem verschiedene epistemische Haltungen indiziert werden (Imo 2009; Barth-Weingarten 2011; Betz/Deppermann 2018).

Auch das Äußerungsende erweist sich als relevante Scharnierstelle für die Kontextualisierung von Diskursrelationen und Wissenszuschreibungen (Haselow 2015): Insbesondere mit question tags etablieren SprecherInnen spezifische Antwortrelevanzen. Zugleich können sie anzeigen, über welchen epistemischen Zugang oder welche epistemischen Rechte sie in Bezug auf den Äußerungsgegenstand verfügen (Raymond/Heritage 2006). Zwar existieren bereits einige Studien, die sich mit einzelnen question tags im Deutschen befassen (Drake 2016; Hagemann 2009; Imo 2011b; Jefferson 1981; König 2017; Willkop 1988), eine systematische Beschreibung, die question tags in einem konstruktionsgrammatischen Rahmen erfasst und die spezifischen interaktionalen Leistungen einzelner tag-Konstruktionen in einem Netzwerk verwandter Konstruktionen verortet, steht bislang jedoch aus.

Hinweis: Für Anmerkungen und Kommentare danke ich den Herausgebern und den TeilnehmerInnen des Workshops „Prosodie und Konstruktionsgrammatik“. Der Beitrag steht in Zusammenhang mit dem von der Deutschen Forschungsgemeinschaft geförderten Netzwerk „Interaktionale Linguistik - Diskurspartikeln aus sprachvergleichender Perspektive“ (Projektnummer 413161127).

Ә Open Access. (c) 2020 Katharina König, publiziert von De Gruyter. (cc) BY Dieses Werk ist lizenziert unter der Creative Commons Attribution-NonCommercial-NoDerivatives 4.0 Lizenz.

https://doi.org/10.1515/9783110637489-006 
Der vorliegende Beitrag wendet sich Gebrauchsmustern mit finalem oder zu, die als teilschematische Konstruktionen - bestehend aus einer schematischen Bezugsäußerung und einem final appendierten, lexikalisch spezifischen oder beschrieben werden sollen. Bisherige Forschungsarbeiten zeigen auf, dass finales oder Unsicherheit der SprecherInnen über das Zutreffen der in der Bezugsäußerung ausgedrückten Proposition zum Ausdruck bringen (Drake 2016; Willkop 1988). Eine genaue Binnendifferenzierung von epistemischen Haltungen, die mit verschiedenen oder-Konstruktionen ausgedrückt werden, liegt bislang jedoch nicht vor. Zudem ist die Rolle der prosodischen Formatierung der Bezugsäußerung und des appendierten oder erst in Ansätzen empirisch erfasst (Drake 2016). Für eine konstruktionsgrammatische Modellierung von Gebrauchsmustern mit finalem oder kann der Einbezug dieses Formmerkmals von zentraler Relevanz sein. Sprachliche Zeichen im Sinne der Konstruktionsgrammatik können von unterschiedlichem Komplexitäts- und Schematizitätsgrad sein (Fillmore et al. 1988; Goldberg 1995); sie sind jedoch stets holistisch zu beschreiben. Während als konsensual gelten kann, dass syntaktische, semantische, pragmatische und sequenzielle Merkmale von Konstruktionen symbolisch miteinander verknüpft sind, ist strittig, ob auf der Formseite eines sprachlichen Zeichens auch prosodische Informationen verfestigt sind (Auer 2016; Ogden 2010) oder ob die prosodische Formatierung bei der Realisierung eines Konstrukts lediglich als Kontextualisierungshinweis hinzutritt, jedoch keinen festen Bestandteil der aus dem konkreten Gebrauch abstrahierten Konstruktion darstellt (Couper-Kuhlen 2012). Am Beispiel von Gebrauchsmustern mit finalem oder soll aufgezeigt werden, dass in dem Zusammenspiel von Bezugsäußerung und tag spezifische prosodische Verfestigungen feststellbar sind, die zur Differenzierung verschiedener Konstruktionen beitragen und somit im Rahmen einer holistischen Konstruktionsbeschreibung mit erfasst werden müssen.

Im Folgenden werden zunächst lexikalische, prosodische, syntaktische und sequenzielle Verfestigungen für Gebrauchsmuster mit finalem oder sowie typische Formatierungen der reaktiven Redezüge beschrieben (Abschnitt 3), um hierauf aufbauend die jeweils kontextualisierte epistemische Haltung zu bestimmen (Abschnitt 4). Durch die Analyse verschiedener Gesprächstypen (Gespräche unter Freuden, Radio-Phone-Ins, qualitative Interviews, Prüfungsgespräche, Unterrichtsinteraktionen) soll zudem ermittelt werden, ob sich die oder-Konstruktionen für die Bearbeitung spezifischer kommunikativer Aufgaben ausdifferenziert haben. Die Befunde können somit auch zur Klärung des Zusammenhangs grammatischer Konstruktionen und kommunikativer Gattungen beitragen (Bücker et al. 2015; Günthner 2006; Imo 2010). 


\section{Befunde zu finalem oder}

In Grammatiken wird oder meist ausschließlich in seiner Verwendung als disjunktive, koordinierende Konjunktion aufgeführt. Hervorgehoben wird etwa, dass oder zur Bildung des Satztyps der Alternativfrage gebraucht wird (Helbig/ Buscha 2013: 616) oder dass es zur Einführung des letzten Elements mehrgliedriger Listen eingesetzt wird (Weinrich 2007: 811). Zwei Lesarten der Disjunktion sind dabei möglich: In der inklusiven Lesart können alle genannten Optionen ausgewählt werden, während in der exklusiven Lesart lediglich ein Alternative gültig sein kann (entweder - oder). Ferner wird laut Hoffmann (2013: 439f.) durch oder eine spezifische epistemische Haltung ausgedrückt: SprecherInnen sind nicht in der Lage, „für einen der Sachverhaltsentwürfe einen Wahrheitsanspruch zu erheben, aber [gehen] davon aus[...], dass wenigstens einer (,inklusives' oder) oder genau einer (,exklusives' oder) wahr ist“.

Finales oder findet etwa bei Hoffmann (2013: 440) und Zifonun et al. (1997: 384) Erwähnung. Es kommt jedoch zu widersprüchlichen Annahmen über die epistemische Haltung, die SprecherInnen gegenüber den Bezugsäußerungen einnehmen. Laut Hoffmann (2013: 440) eröffnet finales oder „die Möglichkeit, das zweite Konjunkt bzw. die zweite Alternative zu ersparen und damit die formulierte Alternative als präferiert, in besonderer Weise erwartet oder als gültig angenommen hinzustellen und zu gewichten“. Diese Auslegung geht also davon aus, dass die bereits verbalisierte Aussage für wahrscheinlich oder zutreffend erachtet wird. Die IDS-Grammatik nimmt eine gegenteilige Präferenz an: „Oder wird nachgeschaltet, wenn der Vergewisserungsbedarf größer ist, der Sprecher also eine Alternative zum assertierten Sachverhalt für möglich oder wahrscheinlich hält“ (Zifonun et al. 1997: 384; vgl. auch Willkop 1988: $271 \mathrm{ff}$.).

Grammatiken erfassen finales oder in seiner Funktion als question tag bzw. Vergewisserungsfrage (Zifonun et al. 1997: 384; Duden 2016: § 883; Weinrich 2007: 833f.). Oft werden jedoch wenige, zumeist dekontextualisierte Beispiele aufgeführt. Angaben über die syntaktische Formatierung der Bezugsäußerung, sequenzielle Kontexte, in die finales oder eingebettet ist, oder sprachliche Handlungen, für die finales oder verwendet wird, erfolgen i.d.R. nicht. Zudem differenzieren Grammatiken nur unzureichend zwischen verschiedenen prosodischen Varianten von finalem oder.

Mit Drake (2016) liegt eine erste Studie vor, die für zwei prosodische Varianten von finalem oder, (final steigender Tonhöhenverlauf) und oder- (final gleichbleibend, trail-off, vgl. Walker 2012, bei Drake als oder_ notiert) unterschiedliche Diskursfunktionen nachweist. Während oder, lediglich zu einer kurzen Bestätigung (oder Ablehnung) der in der Bezugsäußerung ausgedrückten Proposition auffordert (was der Lesart in Hoffmann 2013 entspricht), zeigen 
SprecherInnen durch oder- an, dass sie eine weitere Alternative für möglich erachten (vgl. die Lesart bei Zifonun et al. 1997). Ferner zeigt Drake auf, dass sich die prosodischen Varianten von finalem oder sowohl in Bezug auf die syntaktische Formatierung der Bezugsäußerung als auch in dem Format, das sie als Antwort erwartbar machen, unterscheiden. Ein an Deklarativsätze angehängtes oder, macht eine polare Ja-Nein-Antwort relevant, wobei laut Drake keines der beiden Antwortformate präferiert ist. Mit diesem Gebrauchsmuster werden in Drakes Daten oftmals Nachfragen zum bisher Gesagten gestellt, die im Rahmen kurzer Nebensequenzen geklärt werden. Auf diese Klärung folgt meist ein schneller Themenwechsel. Ein oder-, das sowohl an Interrogativ- als auch an Deklarativsätze angehängt werden kann, lässt hingegen weitere Antworttypen ohne die Responsive wie ja oder nein zu: „oder_turns weaken polar constraints“ (Drake 2016: 180). Mit oder- machen SprecherInnen eine Antwortexplikation relevant, bei der eine Ablehnung der in der Bezugsäußerung ausgedrückten Annahme möglich ist. Finales oder-ist daher oftmals themenexpandierend.

Tab. 1: Befunde zu finalem oder nach Drake (2016).

\begin{tabular}{|c|c|c|}
\hline & oder, & oder- \\
\hline Prosodie des tags & $\begin{array}{l}\text { in die IP integriert, keine eigene } \\
\text { Kontur, keine Akzentuierung, } \\
\text { final steigend }\end{array}$ & $\begin{array}{l}\text { in die IP integriert, i. d. R. keine } \\
\text { eigene Kontur, keine } \\
\text { Akzentuierung, } \\
\text { final gleichbleibend }\end{array}$ \\
\hline $\begin{array}{l}\text { Syntax der Bezugs- } \\
\text { äußerung }\end{array}$ & Deklarativsatz & Interrogativ- oder Deklarativsatz \\
\hline Sequentielle & Anzeige einer & Anzeige einer Verstehensinferenz \\
\hline Einbettung & Verstehensinferenz & \\
\hline $\begin{array}{l}\text { Fortsetzungs- } \\
\text { erwartung }\end{array}$ & $\begin{array}{l}\text { macht J/N-Antwort relevant, } \\
\text { keine eindeutige Präferenz für } \\
\text { Zustimmung oder Ablehnung } \\
\text { schneller Wechsel zu neuem } \\
\text { Thema }\end{array}$ & $\begin{array}{l}\text { Nur schwache Relevantsetzung } \\
\text { einer J/N-Antwort, } \\
\text { Ablehnung der Bezugsäußerung } \\
\text { wird erleichtert } \\
\text { weitere thematische Ausführungen }\end{array}$ \\
\hline $\begin{array}{l}\text { Epistemische Haltung } \\
\text { zur Bezugsäußerung }\end{array}$ & $\begin{array}{l}\text { Relativ sicheres Wissen (im } \\
\text { Vergleich zu oder-) }\end{array}$ & $\begin{array}{l}\text { Relativ unsicheres Wissen (ähnlich } \\
\text { wie finales or-, Drake 2015) }\end{array}$ \\
\hline
\end{tabular}

Beide Gebrauchsmuster mit finalem oder (vgl. Tab. 1) werden laut Drake zur Anzeige einer Verstehensinferenz genutzt (,formulate an understanding“ (Drake 2016: 174, 179). In den Bezugsäußerungen werden mögliche Deutungen der vorausgegangenen Beiträge der GesprächspartnerInnen formuliert; sie dokumentieren 
also gleichzeitig, wie ein vorhergehender Beitrag des Gesprächspartners verstanden wurde, und bieten dieses Verstehen dem Gesprächspartner zur Ratifikation an. Unterschiede können sich jedoch in der epistemischen Haltung zeigen, die mit oder, und oder- gegenüber der Bezugsäußerung zum Ausdruck gebracht wird. Drake (2016) zufolge weist finales oder- Ähnlichkeiten zu finalem or- im amerikanischen Englisch auf, das eine relativ hohe Sprecherunsicherheit gegenüber der Bezugsäußerung kontextualisiert, da es eine weitere Alternative andeutet, eine unproblematische Ablehnung ermöglicht und eine elaborierte Reaktion erwartbar macht (Drake 2015: 312ff.). Da oder, diese Funktionen nicht aufweist, müsste geschlossen werden, dass es im Gegensatz zu oder- eine sicherere epistemische Haltung anzeigt. Eine Diskussion über einen epistemischen Gradienten zwischen oder, und oder- erfolgt bei Drake jedoch nicht.

Im vorliegenden Beitrag soll daher eruiert werden, ob und wie die Annahme eines epistemischen Gefälles zwischen den beiden oder-Varianten aus den Daten hergleitet werden kann. Der Artikel baut somit auf den Befunden Drakes auf und geht auf Basis von Gesprächsdaten aus verschiedenen Interaktionstypen der Frage nach, ob Konstruktionen mit finalem oder auf verschiedene Handlungskontexte zugeschnitten sind. Dabei soll die Rolle der lexikalischen, syntaktischen und prosodischen Formatierung der Bezugsäußerung ebenso reflektiert werden wie die Einbettung der Bezugsäußerungen in den vorhergehenden sequenziellen Kontext. Ferner sollen die Befunde dahingehend systematisiert werden, dass die präferentielle Ordnung der Antwortalternativen in verschiedenen oder-Konstruktionen untersucht wird. Auf Basis dieser Analysen werden vier Form-Funktions-Paare mit finalem oder differenziert, die sich insbesondere in der jeweils ausgedrückten epistemischen Haltung unterscheiden.

\section{Konstruktionen mit finalem oder}

Die Untersuchung basiert auf einer Kollektion von 41 Vorkommen von Äußerungen mit finalem Oder, ${ }^{1}$ und 58 Vorkommen mit finalem Oder-, die anhand von 77 Gesprächen erstellt wurde, von denen ca. 18 Stunden transkribiert vorliegen. Als Gesprächstypen sind neben privaten Alltagsgesprächen zwischen FreundInnen und Familienmitgliedern (Telefongespräche und Tischgespräche) auch sprachbiographische Interviews und Radio-Phone-Ins als institutionelle

1 Um hervorzuheben, dass der Fokusakzent bei appendiertem Oder stets auf der ersten Silbe liegt, wird die Akzentsilbe im Folgenden mit einer Majuskel notiert. 
bzw. medial vermittelte, öffentliche Interaktionen repräsentiert. ${ }^{2}$ Die Daten entstammen der linguistischen Audio-Datenbank (lAuDa), dem Korpus Multimodaler Interaktion (KoMI), dem Interviewkorpus des Projekts Sprachvariation in Norddeutschland sowie dem Lehrkorpus Sprachbiographien. ${ }^{3}$ Darüber hinaus wurden zur Untersuchung von Prüfungs- und Unterrichtsgesprächen als weiteren institutionellen Interaktionstypen Daten im Umfang von etwa sechs Stunden aus dem Forschungs- und Lehrkorpus (FOLK) herangezogen, in denen 17 Belege von finalem !O!der, identifiziert wurden. ${ }^{4}$

Die Transkription erfolgt nach den GAT2-Konventionen (Selting et al. 2009). Es zeigt sich, dass - anders als in den von Drake (2016) untersuchten Daten - in der Regel sowohl die Bezugsäußerung als auch das angehängte oder eigenständige Intonationsphrasen bilden. Informationen über finale Tonhöhenbewegungen werden im Folgenden daher sowohl für die Bezugsäußerung als auch für das appendierte oder erfasst. Prototypischerweise folgen Oder, und Oder- im schnellen Anschluss an die vorhergehende Äußerung (Abb. 1 und 2); sie können jedoch auch durch Pausen (und teilweise auch durch Sprecherwechsel)



Abb. 1: $\left[\mathrm{X}_{\mathrm{V} 2} ;=\right.$ Oder, $]$ aus Beispiel (1).

2 Die hier präsentierten Befunde beziehen sich auf Verwendungsmuster von finalem oder im norddeutschen Sprachraum. Dass sich in anderen Sprachgebieten unterschiedliche Verwendungsweisen von finalem oder vorliegen können, belegt etwa die Untersuchung Glasers (2003) zu finalem oder im Berndeutschen.

3 Für weitere Angaben zur lAuDa siehe http://lauda-ms.lingdata.de/. Für die Bereitstellung der Daten des KoMI danke ich Pepe Droste. $\mathrm{Zu}$ den Daten des SiN-Interviewkorpus siehe https://www.corpora.uni-hamburg.de/sin/startseite.html. Informationen zu dem Lehrkorpus Sprachbiographien sind der folgenden Webseite zu entnehmen: https://www.uni-muenster.de/ Germanistik/Lehrende/sprachwissenschaft/koenig_katharina/lehrkorpus.html.

4 Die FOLK-Daten sind über die Datenbank Gesprochenes Deutsch am Institut für Deutsche Sprache abrufbar: https://dgd.ids-mannheim.de. 


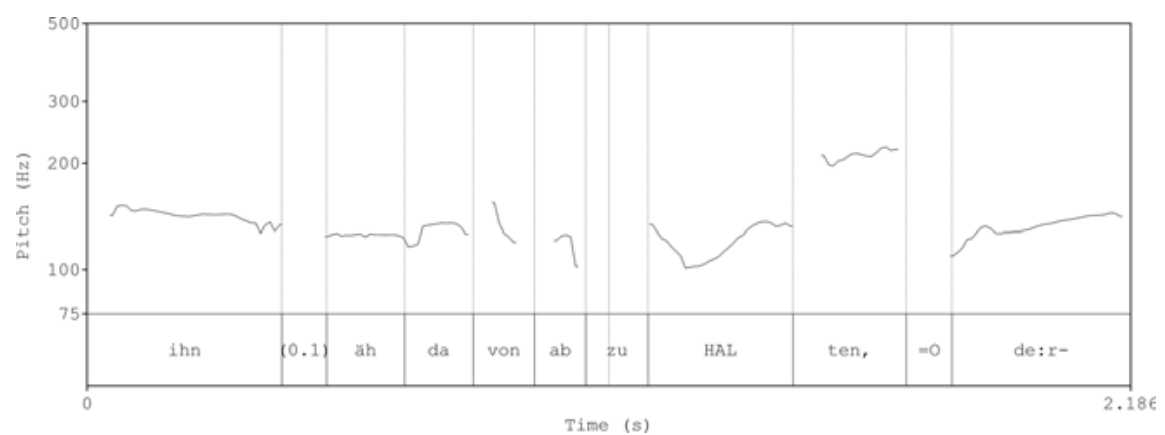

Abb. 2: $\left[\mathrm{X}_{\mathrm{V} 2},=\right.$ Oder- $]$ aus Beispiel (4).

von der Bezugsäußerung abgesetzt sein. Es kommt also zu unterschiedlich starken Zäsurierungen (Barth-Weingarten 2016). Finales Oder-ist meist durch einen tiefen Tonhöhenansatz von der vorherigen Intonationsphrase abgesetzt; ferner ist die erste Silbe i.d.R. in Knarrstimme realisiert. In einigen Fällen kommt es zudem zu einer Dehnung des Vokals der zweiten Silbe. Auch finales !O!der, (Abb. 3) bildet eine eigene Intonationsphrase; der finale Tonhöhenverlauf ist (leicht) steigend. Die erste Silbe ist hingegen i.d.R. durch einen tiefen Tonhöhenansatz abgesetzt und durch starke Akzentuierung hervorgehoben.

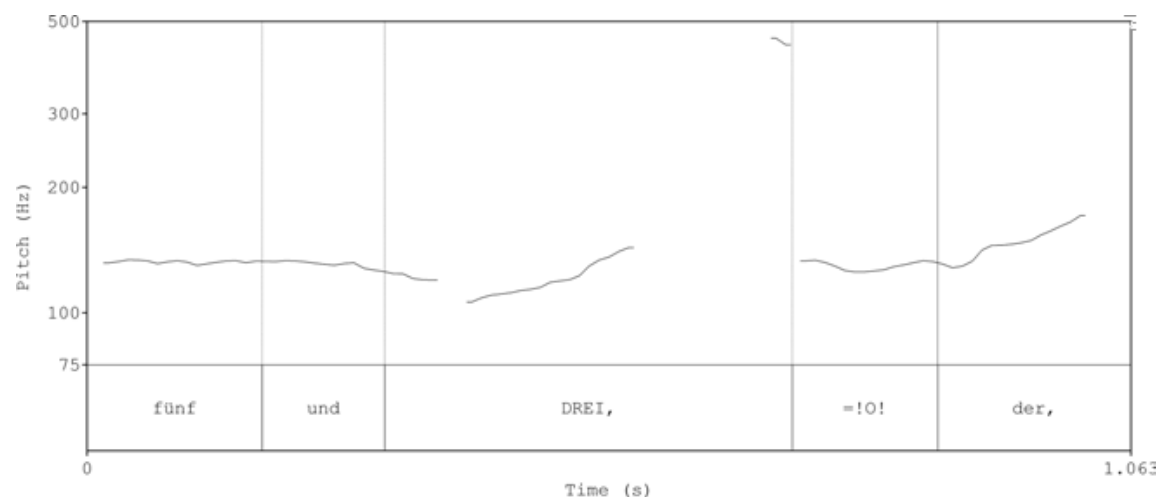

Abb. 3: $\left[\mathrm{X}_{\mathrm{V} 2 / \text { Fragment }}\right.$, OO!der, $]$ aus Beispiel (7).

Im Folgenden werden Vorkommen von finalem oder stets im Zusammenspiel mit der vorhergehenden Äußerung betrachtet, die sich im Skopus von oder befindet. Es zeigt sich, dass die in der Kollektion nachgewiesenen prosodische Varianten von finalem oder (Oder, sowie Oder- und !O!der,) mit jeweils spezifisch formatierten Bezugsäußerungen auftreten und sich für die Kombinationen aus 
Bezugsäußerung und tag verschiedene Bedeutungspotenziale nachweisen lassen. Daher ist von teilschematischen Form-Funktions-Paarungen auszugehen.

\section{$3.1\left[\mathrm{x}_{\mathrm{v} 2}=\right.$ Oder, $]$}

Die erste Konstruktion erweist sich als hochgradig stabil in der prosodischen Formatierung: Alle 41 Vorkommen von oder, in der Kollektion haben einen Verbzweitsatz als Bezugsäußerung; in allen Fällen ist die Bezugsäußerung mit fallender oder leicht fallender finaler Tonhöhenbewegung realisiert. Das Oder, wird in einer schnell angeschlossenen, eigenständigen Intonationsphrase mit final (leicht) steigender Tonhöhenbewegung appendiert. Das folgende Beispiel aus einem Skype-Telefonat zweier Freundinnen präsentiert eine prototypische Verwendungsweise dieses Musters. Dana befindet sich gerade für ein Auslandssemester in Kanada und berichtet von einem Besuch in einem Naturkundemuseum, bei dem sie zahlreiche Exponate von kanadischen Wildtieren erkunden konnte.

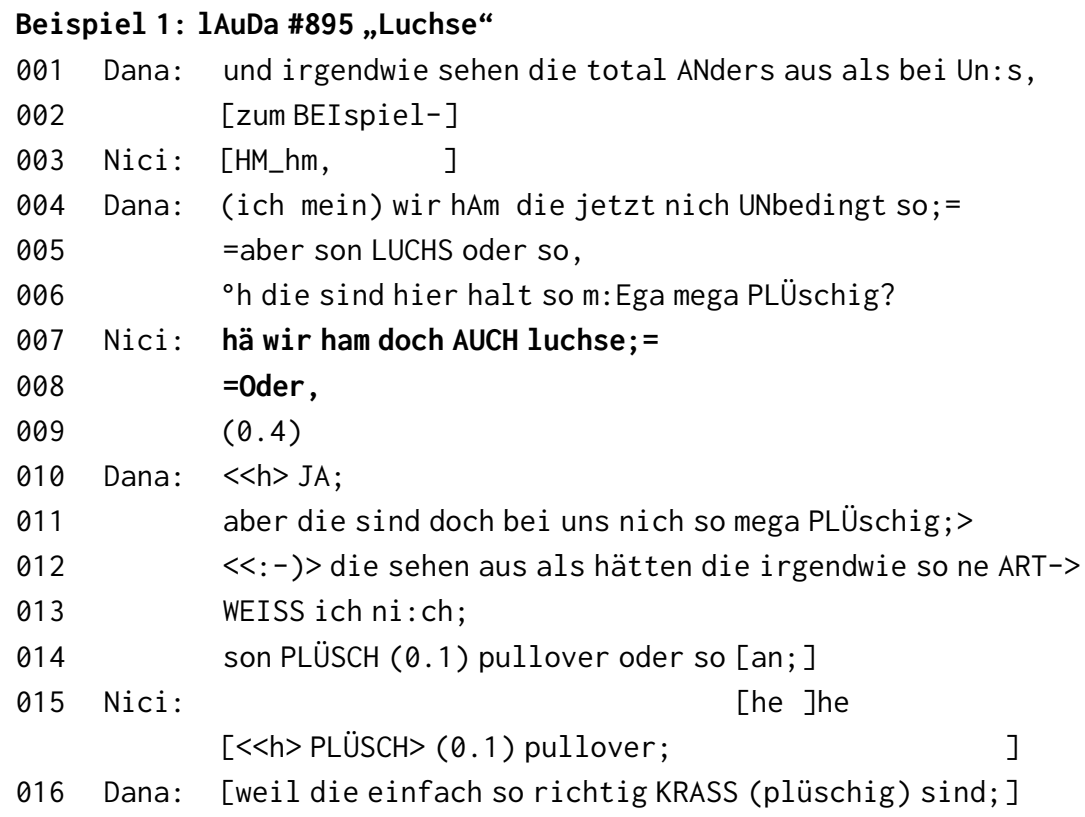


In Nicis Nachfrage in 007 dokumentiert sich ein thematischer Anschluss an den vorherigen Gesprächskontext: Sie formuliert den Einwand, dass Luchse in Europa (im Gegensatz zu Nordamerika) ebenfalls heimisch seien. Durch die Gesprächspartikel hä sowie die Modalpartikel doch ist die ausgedrückte Annahme als Widerspruch markiert. ${ }^{5}$ Nici rahmt die Äußerung auf diese Weise als eine Proposition, von deren Gültigkeit sie bislang überzeugt war. Auch wenn sie mit ihrem Einwand ein Verstehen des vorher Gesagten dokumentiert (sie unterstellt Dana die Annahme, dass es in Europa keine Luchse gibt, vgl. Danas Äußerung in 004) und zugleich mögliche Präsuppositionen von Danas Ausführungen überprüft (vgl. Selting 1995: 268), ist festzuhalten, dass die in dem Einwand ausgedrückte Proposition selbst nicht aus dem bisher Gesagten inferiert wird, sondern in einer gesprächsunabhängigen Wissensdomäne verortet ist.

Bestätigungen von mit oder, appendierten final fallenden Verbzweitsätzen werden wie hier oftmals durch ein einfaches responsives ja realisiert. Es folgen keine oder nur kurze Antworterläuterungen; wie Drake (2016: 179) bereits konstatiert, leiten SprecherInnen schnell zum nächsten Handlungsschritt über (in diesem Fall weitere Ausführungen zum Phänotyp kanadischer Luchse). Die eigentliche Bestätigung der zur Ratifikation angebotenen Aussage in 007 erfolgt also als schnell abgehandelte Nebensequenz.

Anders gestalten sich hingegen Ablehnungen der in der Bezugsäußerung ausgedrückten Annahmen. In einem Telefongespräch berichtet Jenny ihrer Freundin Silke von Problemen, die sie mit den hohen Sprachanforderungen ihres Anglistik-Studiums hat.

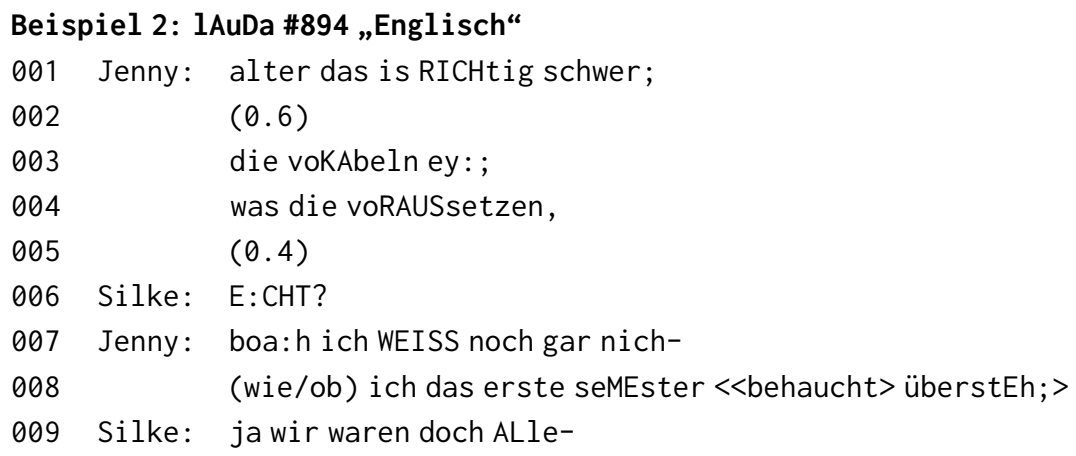

$5 \mathrm{Zu}$ doch als Modalpartikel siehe auch Diewald/Fischer (1998: 92f.). In der Grundbedeutung der Partikel kommt laut Diewald/Fischer die Existenz einer möglichen Alternative zum Ausdruck. Der Sprecher/die Sprecherin ist aber der Überzeugung, dass die verbalisierte Proposition gilt. 


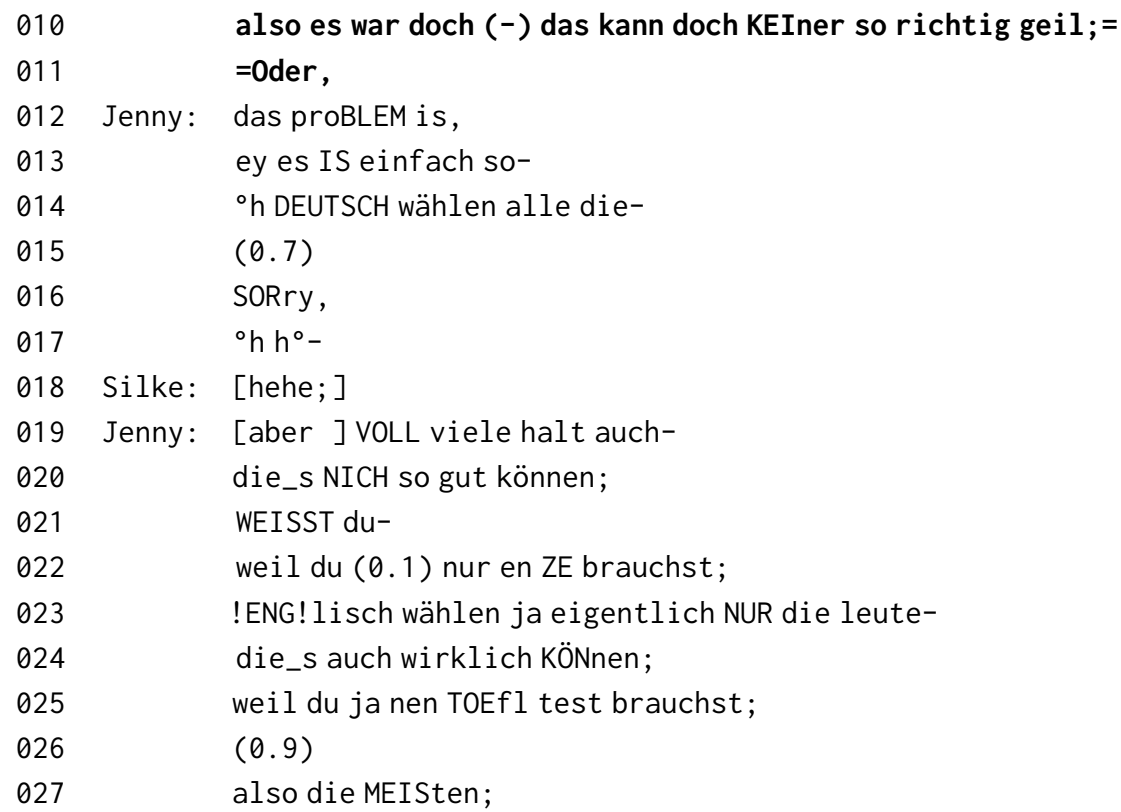

Auch in diesem Beispiel stellt die zur Ratifikation angebotene Aussage zwar eine thematische Fortführung des zuvor etablierten Gesprächsgegenstands dar, sie bezieht sich jedoch erneut nicht auf bereits in das Gespräch eingeführte Wissensbestände. Vielmehr nimmt Silke eine Charakterisierung der Gruppe der AnglistikStudierenden vor, der sie selbst nicht angehört. Mit der Partikel doch findet sich abermals eine Modalisierung als Widerspruch zu Jennys bisherigen Ausführungen. Durch das finale Oder, wird ein herabgestufter epistemischer Zugang angezeigt, der zugleich die Direktheit des Widerspruchs abschwächen kann. ${ }^{6}$

In der Folge bestätigt Jenny Silkes Annahme, dass Anglistik-Studierende die Sprache nicht gut beherrschen, nicht, sondern bringt eine gegenteilige Einschätzung zum Ausdruck (023-024). Die Zurückweisung von Silkes Bewertung erfolgt dabei in einem indirekten Format: Die Projektor-Konstruktion „das Problem ist“ wird als verzögerndes Vorlaufelement eingesetzt (vgl. Imo 2010), bevor Jenny schließlich ihre Einschätzung verbalisiert, die zudem noch durch eine Begründung expandiert wird. Anders als im ersten Beispiel wird die Bearbei-

6 Eine ähnliche Funktion lässt sich auch für Beispiel 1 annehmen. 
tung des nächsten Handlungsschritts hierdurch verzögert. Dies deutet darauf hin, dass die Antwortalternativen nach einer Äußerung mit appendiertem Oder, entgegen der Befunde Drakes präferenziell geordnet sind: Während eine Zustimmung problemlos erfolgen kann, ist eine Zurückweisung der Äußerung als dispräferiert markiert. Zwar finden sich in der Kollektion nicht viele Ablehnungen (12/41) von mit Oder, appendierten Annahmen, jedoch sind diese in zehn Fällen durch Pausen, Zögerungen oder Vorlaufelemente (wie etwa Projektorkonstruktionen) als dispräferiert gerahmt.

In Äußerungen mit appendiertem Oder, verbalisieren SprecherInnen i.d. R. Annahmen, die sich nicht aus dem unmittelbaren Gesprächskontext inferieren lassen (und mitunter sogar im Gegensatz zu Präsuppositionen des zuvor Gesagten stehen), derer sie sich aber relativ sicher sind (u. a. angezeigt durch die Wahl eines deklarativen Verbzweitsatzes sowie die fallende Kontur der Bezugsäußerung). ${ }^{7}$ Durch finales Oder, bieten SprecherInnen die Bezugsäußerung ihren GesprächspartnerInnen aber zur intersubjektiven Ratifikation an, wodurch sie wiederum eine epistemische Herabstufung vornehmen. ${ }^{8}$ Es werden also keine weiteren Antwortmöglichkeiten in Aussicht gestellt, sondern es soll lediglich die Gültigkeit der Bezugsäußerung überprüft werden (Gilt X oder $\neg X$ ?). ${ }^{9}$ In ihren Reaktionen behandeln SprecherInnen Zustimmungen daher als präferiert, Ablehnungen hingegen als dispräferiert.

\section{$3.2\left[\mathrm{X}_{\mathrm{V} 2,}=\right.$ Oder -$]$}

Auch mit Deklarativsätzen, an die ein Oder- angehängt wird, bieten SprecherInnen ihren GesprächspartnerInnen eine Proposition zur Ratifikation an. Bei dieser Konstruktion zeigen sich jedoch zum einen andere lexikalische, prosodische und sequenzielle Verfestigungen und zum anderen von dem ersten Muster abweichende epistemische Bedeutungspotenziale. Von den 58 Vorkommen von finalem Oder- in der Kollektion sind 21 an einen vorhergehenden Verbzweitsatz appendiert. Dieser ist - im Unterschied zu der in 3.1. beschriebenen

7 Zum möglichen epistemischen Gradienten zwischen Deklarativ- und Interrogativsätzen siehe Heritage (2013: 378), und Deppermann (2015). Zum epistemischen Kontextualisierungspotenzial der prosodischen Formatierung von V2-Deklarativsätzen vgl. Selting (1995), für ähnliche Befunde im Englischen siehe Couper-Kuhlen (2012).

8 Diese Herabstufung kann sowohl den Wissenszugang (epistemic access) als auch die Wissensrechte (epistemic rights, etwa bei Bewertungen) betreffen.

9 Vermittels dieser Positionierung kann auch ein ggf. in der Bezugsäußerung ausgedrückter Widerspruch abgemildert werden. 
Konstruktion - nur in fünf Fällen mit final fallender Kontur realisiert. ${ }^{10}$ Bei den meisten Vorkommen findet sich eine leicht steigende finale Tonhöhenbewegung. Anhand der folgenden Beispiele sollen prototypische sequenzielle Einbettungen und Antwortpräferenzen aufgezeigt werden.

Beispiel 3 ist einem Radio-Phone-In entnommen, bei dem der Anrufer Andreas (AS) berichtet hat, dass er sich in seine beste Freundin verliebt hat, die derzeit jedoch noch in einer anderen Beziehung lebt. Der Moderator Benjamin (BJ) stellt nun Nachfragen zu der Freundschaft.

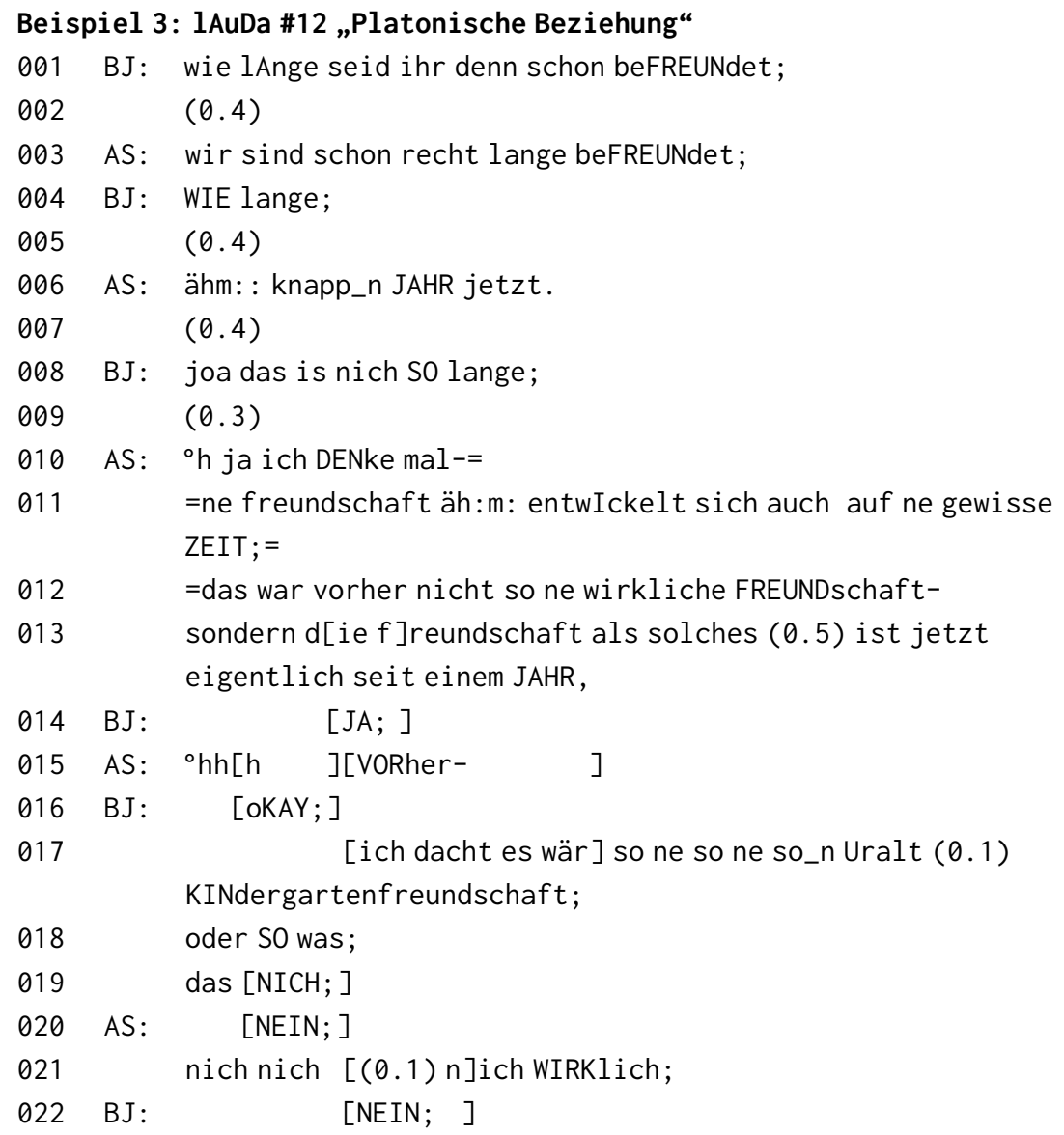

10 Bei fünf Vorkommen liegt eine gleichbleibende finale Formatierung vor, wobei es sich bei diesen Belegen um Bezugsäußerungen im Kontext einer mindestens zwei Elemente umfassenden Auflistung handelt (vgl. Selting 2004). Durch diese Einbettung wird bereits eine potenzielle Fortführbarkeit, also die Möglichkeit einer weiteren Alternative, kontextualisiert. 


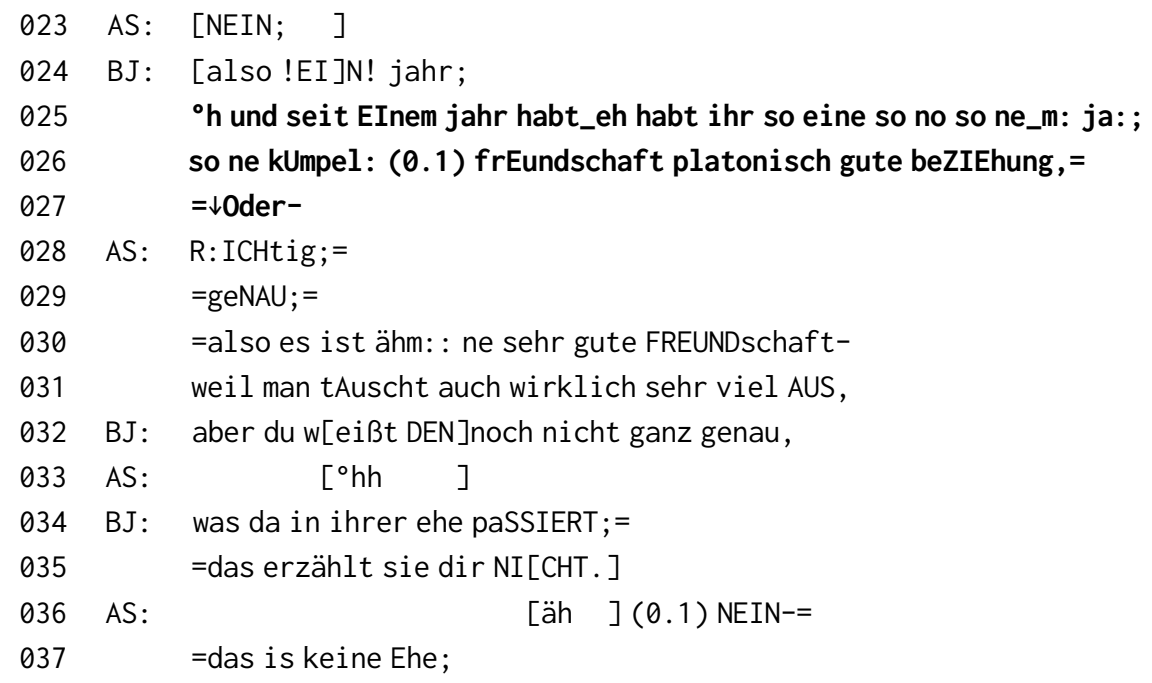

In Segmenten 025-026 bietet Benjamin Andreas eine mögliche Charakterisierung der Freundschaftsbeziehung an. Anders als bei der in Abschnitt 3.1 vorgestellten Konstruktion ist die in der Bezugsäußerung verbalisierte Proposition aus Andreas' vorherigen Ausführungen inferiert. Bereits in Segment 017 hat Benjamin sein Verständnis des bisherigen Gesprächs dokumentiert. Im thematischen Anschluss, durch das initiale und kontextualisiert, setzt er diese Inferenzdokumentation fort und verbalisiert eine Proposition, die ihm für das Nachvollziehen von Andreas' Situation in der vorherigen Darstellung fehlt (siehe auch Bolden 2010).

In dem Format eines Verbzweitsatzes mit final steigender Tonhöhenbewegung ist Benjamins Inferenz bereits als offene Frage realisiert, die eine elaborierte Antwort relevant macht (vgl. Selting 1995: 252). Zudem ist die Inferenzdarstellung durch Wiederholungen, Reparaturen und Neuansätze als Suche nach einer passenden Formulierung gerahmt, was in dem gegebenen Kontext ebenfalls zur Anzeige einer relativen Unsicherheit in Bezug auf die Passung der Formulierung dient. Partikeln, die eine relativ sichere epistemische Haltung dokumentieren (wie etwa doch in Beispiel 1), finden sich in dieser Konstruktion nicht. Schließlich rahmt das appendierte Oder- (027) die Kategorisierung nachträglich als eine von weiteren möglichen Bezeichnungsalternativen. Sowohl die disjunktive Semantik der Konjunktion oder als auch die final gleichbleibende Kontur und die Dehnung kontextualisieren eine potenzielle Fortführbarkeit. Anders als dies etwa bei Aposiopesen der Fall ist (Imo 2011a), wird die insinuierte Alternative nicht als zwischen den Interagierenden geteiltes Wissen behandelt; vielmehr obliegt es der 
adressierten Person, über das Zutreffen der verbalisierten Proposition zu urteilen oder ggf. eine bislang noch nicht verbalisierte Alternative zu präsentieren.

Da eine weitere Möglichkeit in Aussicht gestellt wird, erfolgt die Bestätigung der Passung der Beziehungscharakterisierung mit sprachlichen Ausdrücken, die gegenüber einem einfachen ja als starke Zustimmung markiert sind: Mit den Responsiven „RICHtig;“ und „geNAU“; vollzieht Benjamin mehr als nur eine Passungsbestätigung. Er unterstreicht die uneingeschränkte Gültigkeit der vorherigen Aussage sowie seinen primären epistemischen Zugang $\mathrm{zu}$ dem Gegenstand (vgl. Oloff 2017), der allein in seinem biographischen Wissen verortet ist. In Segmenten 030 und 031 beginnt Andreas zudem eine erläuternde Ausführung, die prosodisch eine Weiterführung erwartbar macht, jedoch durch den Moderator unterbrochen wird. SprecherInnen betreiben somit auch bei einer Zustimmung nach einer $\left[\mathrm{X}_{\mathrm{V} 2},=\right.$ Oder- $]$-Konstruktion einen höheren konversationellen Aufwand als bei $\operatorname{der}\left[\mathrm{X}_{\mathrm{V} 2} ;=\right.$ Oder, $]$-Konstruktion.

Beispiel 4, das ebenfalls aus einem Radio-Phone-In stammt, illustriert den Umgang mit einer Zurückweisung der in der Bezugsäußerung verbalisierten Proposition. Die Anruferin Christina berichtet davon, dass ihr Freund als Soldat in den Afghanistan-Krieg ziehen will und sich daher von ihr getrennt hat.

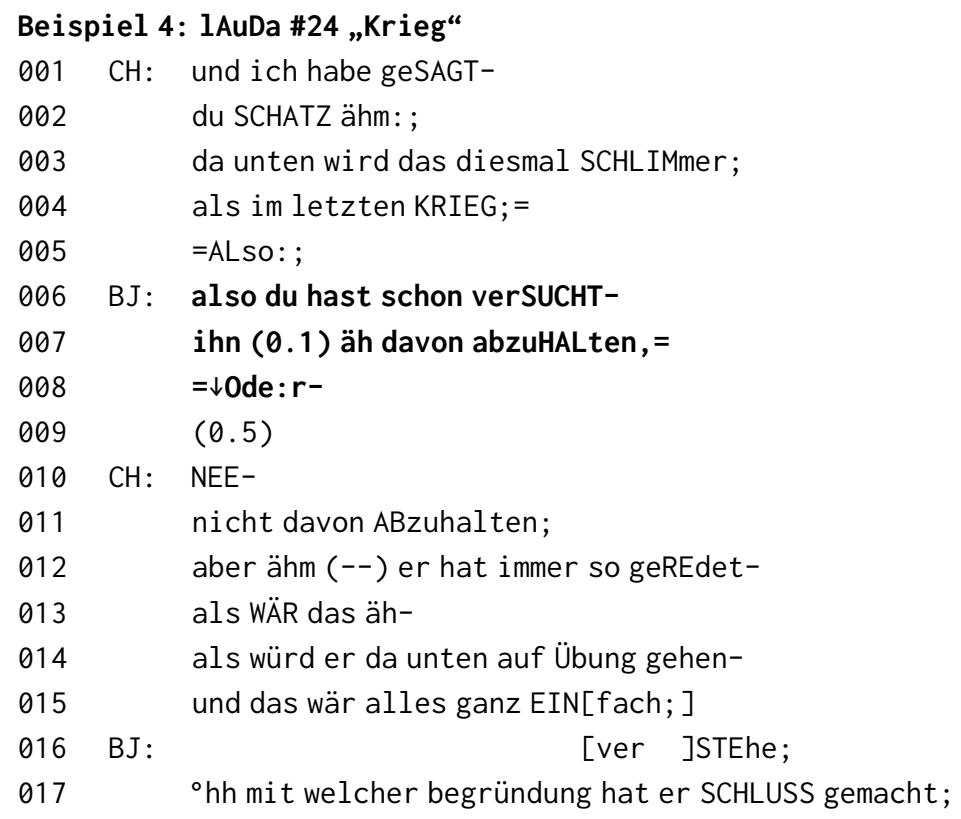


In diesem Ausschnitt stellt der Sprecher die Äußerung in 006-008 ebenfalls als Inferenz aus dem zuvor Gesagten dar (angezeigt durch den im Vor-Vorfeld positionierten Konnektor also, vgl. Deppermann/Helmer 2013). Die Inferenz wird Christina zur Ratifikation bzw. zur weiteren Kommentierung vorlegt. Auch hier kontextualisieren Pausen und andere Zögerungssignale, die final steigend formatierte Bezugsäußerung wie auch das gedehnte „Ode:r-“ (008), das eine weitere mögliche Alternative andeutet, einen herabgestuften epistemischen Zugang des Moderators.

Anders als in Abschnitt 3.1 skizziert wurde, erfolgen Ablehnungen nach der $\left[\mathrm{X}_{\mathrm{V} 2,}=\right.$ Oder-]-Konstruktion oftmals in einem direkten und nicht oder nur minimal als dispräferiert markierten Format. Auf das kurz verzögerte negative Responsiv „nee“ (010) erfolgt in dem gegebenen Beispiel eine negierte Wiederholung der von dem Moderator eingebrachten Formulierung. Im nächsten Segment reformuliert Christina ihre Kritik an der Perspektive, die ihr Freund auf den Krieg einnimmt. Der Account wird jedoch nicht weiter ausgebaut, da Benjamin schnell Verstehen signalisiert und zu einer Nachfrage ansetzt (016-017).

Die Reaktionen auf Verbzweitsätze mit finalem Oder-, nach denen ein Sprecherwechsel erfolgt, sind überwiegend mit Responsiven (genau, richtig, nee, nein, nö) realisiert. ${ }^{11}$ Anders als Drake konstatiert, hebt Oder- also nicht in allen Fällen die Verpflichtung zu einem polaren Antwortformat auf. Vielmehr bleibt die Präferenz für Ja/Nein-Antworten bei $\left[\mathrm{X}_{\mathrm{V} 2},=\right.$ Oder-]-Konstruktionen erhalten. Jedoch ist die eindeutige Präferenz für zustimmende Handlungen aufgehoben. Ablehnungen und Zustimmungen sind in der Kollektion weitgehend gleich gestaltet. Bei beiden Handlungen sind Accounts für die (Nicht-)Passung der Inferenzdokumentationen festzustellen. Dieses Reaktionsverhalten kann als Hinweis dafür gedeutet werden, dass die SprecherInnen mit Oder- appendierte Äußerungen als epistemisch herabgestufter behandeln als durch Oder, appendierte Äußerungen.

\section{$3.3\left[\mathrm{X}_{\mathrm{v} 1}=\right.$ Oder -$]$}

Am häufigsten findet sich finales Oder- in der Kollektion in Bezug auf eine final steigend formatierte Verberstfrage $(27 / 58){ }^{12}$ Bei dieser Konstruktion nun bestätigt sich der Befund Drakes, dass mit dem finalen oder- die Verpflichtung zu einer für

11 In acht von elf Fällen werden Responsive vor den Accounts platziert.

12 Nur ein Vorkommen einer $w$-Verb-Zweit-Frage mit finalem oder-ist belegt. Vorkommen von oder- im Kontext von Alternativfragen (9 Belege) werden hier nicht ausgewertet. $\mathrm{Zu}$ den 21 Vorkommen mit Verbzweitsätzen siehe Abschnitt 3.2. 
Verberstfragen charakteristischen binären Ja-Nein-Antwort gelockert wird. In dem folgenden Ausschnitt aus einem sprachbiographischen Interview wird das Format von der Interviewerin realisiert.

\section{Beispiel 5: Lehrkorpus \#8 „Großeltern“}

001 Ugur: meine Eltern fahren: (-) eigentlich nur in die türKEI; =

$002 \quad=$ zum BEIspiel.

003 Int: hm_[HM, ]

004 Ugur: [WEIL se halt-]

005 da haben sie ihre faMIlie noch-

006 Int: [hm_HM, ]

007 Ugur: [nen BISSchen-]

$008 \quad{ }^{\circ}$ hh die geht man beSUchen-

009 und (0.1) JA.

010 Int: HM;

011 leben denn deine GROSSeltern noch,

$012 \quad=<<$ p $>\downarrow$ Oder $->$

012 Ugur: $\ddot{\mathrm{AH}}$ : ;

$013 \quad(0.4)$

014 meine Oma?

015 also_äh die mutter meines VAters-=

$017=$ ähm: wohnt HIER,

018 Int: [hm_HM, ]

019 Ugur: [bei UNS, ]

020 in DEUTSCHland,

021 direkt tür and TÜR wohnen wir,

022 Int: [AH ja; ]

023 Ugur: [SO, ]

024 Int: [oKAY; ]

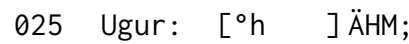

$026 \quad<<$ creaky>mh>;

027 die eltern meiner (0.1) mutter wohnen in der türKEI noch-

028 un:d $\ddot{A} H M-$

$029 \quad(0.4)$

030 Int: [HM; ]

031 Ugur: [ ${ }^{\circ} \mathrm{h}$ ja ] dann gibt_s halt regelmäßig telefoNAte-

032 und dann wird halt auch geSPROchen-= 


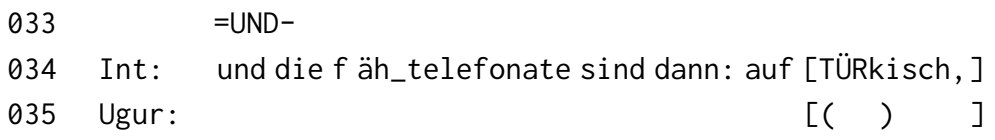

Die Verwendung der Partikel denn ist charakteristisch für das vorliegende Verwendungsmuster, in dem oftmals Verstehensinferenzen zum Ausdruck gebracht werden (vgl. Deppermann 2009). Die in 011 formulierte Frage wird so als Nachfrage gerahmt, die sich für die Interviewerin aus den vorherigen Ausführungen des Interviewpartners Ugur ergibt. Zum einen dokumentiert die Frage also ein Verstehen des bisher Gesagten (Familienbesuche) und verpflichtet den Adressaten Tim zum anderen auf eine Präzisierung der vorherigen Aussage (es soll spezifiziert werden, ob als Familienbesuche auch Besuche bei den Großeltern möglich sind). Neben der Partikel denn werden in den untersuchten Daten ebenso initiales und oder andere additive Ausdrücke wie auch oder sonst noch gebraucht, um die Frage explizit als Anschlussfrage zu markieren. In nur sechs der 27 Vorkommen der Konstruktion erfolgt keine solche Anschlussmarkierung auf der sprachlichen Oberfläche. Ferner deutet sich an, dass Oder- mit bestimmten Wissensbeständen verbunden wird: Wie auch in Beispielen 3 und 4 ist es hier abermals biographisches Wissen, das mit einer durch Oder- appendierten Äußerung abgefragt wird.

In seiner Reaktion auf die Frage der Interviewerin stimmt Ugur der in der Frage ausgedrückten Annahme zu, allerdings ohne confirmation token. Die Zustimmung wird vielmehr implizit in einer längeren thematischen Ausführung vollzogen, in der die genauen Lebensumstände der Großeltern dargelegt werden. Insgesamt sind in der Kollektion nur acht Responsive als Antwortformat bei dieser Konstruktion dokumentiert; die Bestätigung oder Ablehnung der in der Frage ausgedrückten Inferenz erfolgt also überwiegend durch expandierende Ausführungen.

Das folgende Beispiel aus einem WG-Gespräch zeigt, dass SprecherInnen eine explizite Ablehnung der in der Bezugsäußerung ausgedrückten Proposition ohne Dispräferenzmarker realisieren können.

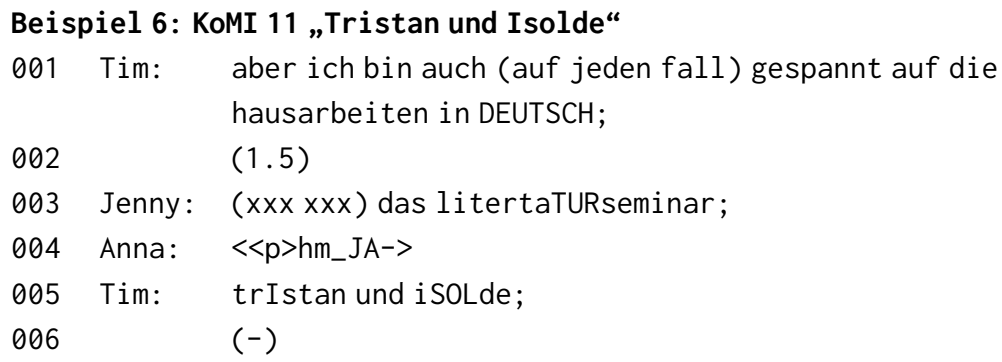




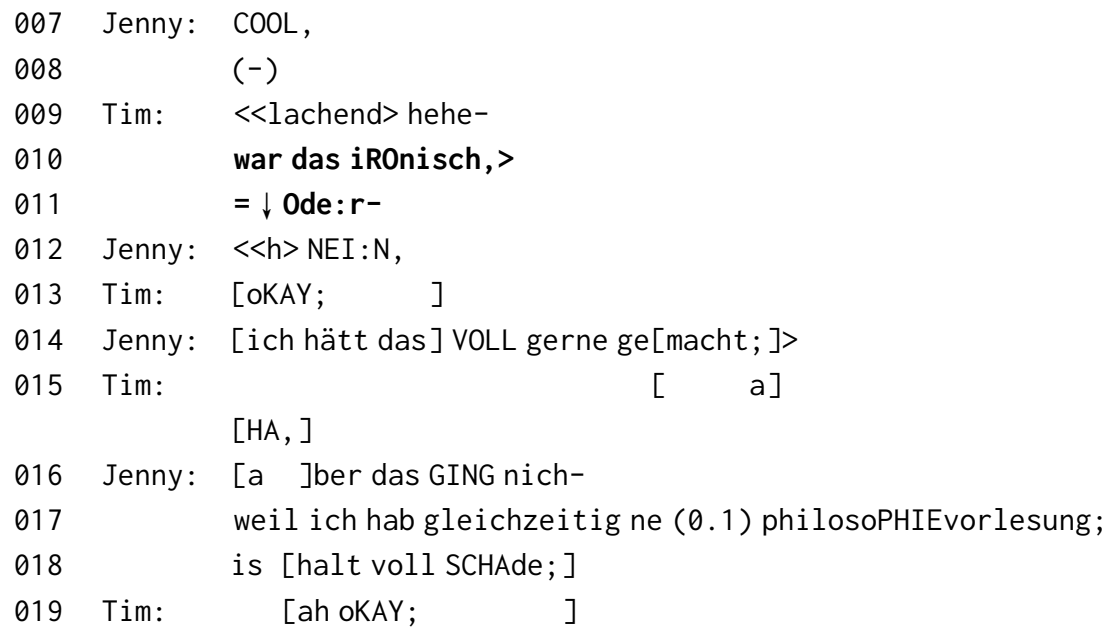

Wie bereits im vorherigen Beispiel wird auch in diesem Ausschnitt eine Nachfrage gestellt, die sich für Tim aus dem bisherigen Gesprächskontext ergibt. ${ }^{13}$ Der Ausschnitt belegt, dass eine direkte Ablehnung der in der Bezugsäußerung ausgedrückten Annahme vermittels eines negativen Responsivs ohne Dispräferenzmarker wie Verzögerung oder Vorlaufelemente möglich ist. Das nein ist durch die Dehnung und den Wechsel in eine höhere Stimmlage sogar prosodisch herausgestellt. Auch in diesem Fall erfolgt eine Ausführung über die Gründe der Ablehnung. Sowohl bei zustimmenden als auch bei ablehnenden Reaktionen auf Verberstfragen macht finales Oder- also themenexpandierende Elaborationen relevant.

\section{$3.4\left[\mathrm{X}_{\mathrm{V} 2 / \text { Fragment, }} ! 0 !\right.$ der, $]$}

Finales oder wurde in allen der bisher diskutierten Verwendungsweisen als Marker für ein epistemisches downgrading verwendet, das sich für die Durchführung verschiedener Handlungen in den untersuchten Gesprächskontexten als funktional erweist (etwa für die kurze Verhandlung konträrer Annahmen oder für erzählgenerierenden Nachfragen). In den ausgewerteten Prüfungsund Unterrichtsgesprächen findet sich eine weitere Verwendungsweise von

13 Auch hier wird Wissen thematisiert, über das einzig die adressierte Person selbst verfügen kann. 
finalem oder die unter von den bisherigen Konstruktionen abweichenden epistemischen Grundbedingungen operiert.

Das folgende Beispiel aus dem Forschungs- und Lehrkorpus (FOLK) entstammt einer Unterrichtsinteraktion einer Berufsschulklasse, die sich mit der Funktionsweise von Ottomotoren befasst. Der Lehrer Lukas Bade (LB) hat im Voraus eine Frage nach den Prüfbedingungen gestellt, die gegeben sein müssen, um eine bestimmte Spannung abzulesen. Der Schüler Jason Mendel (JM) hat diese Frage bereits in Teilen richtig beantwortet (vgl. die Bewertung in 001). Ab Segment 003 setzt JM seine Antwort fort.

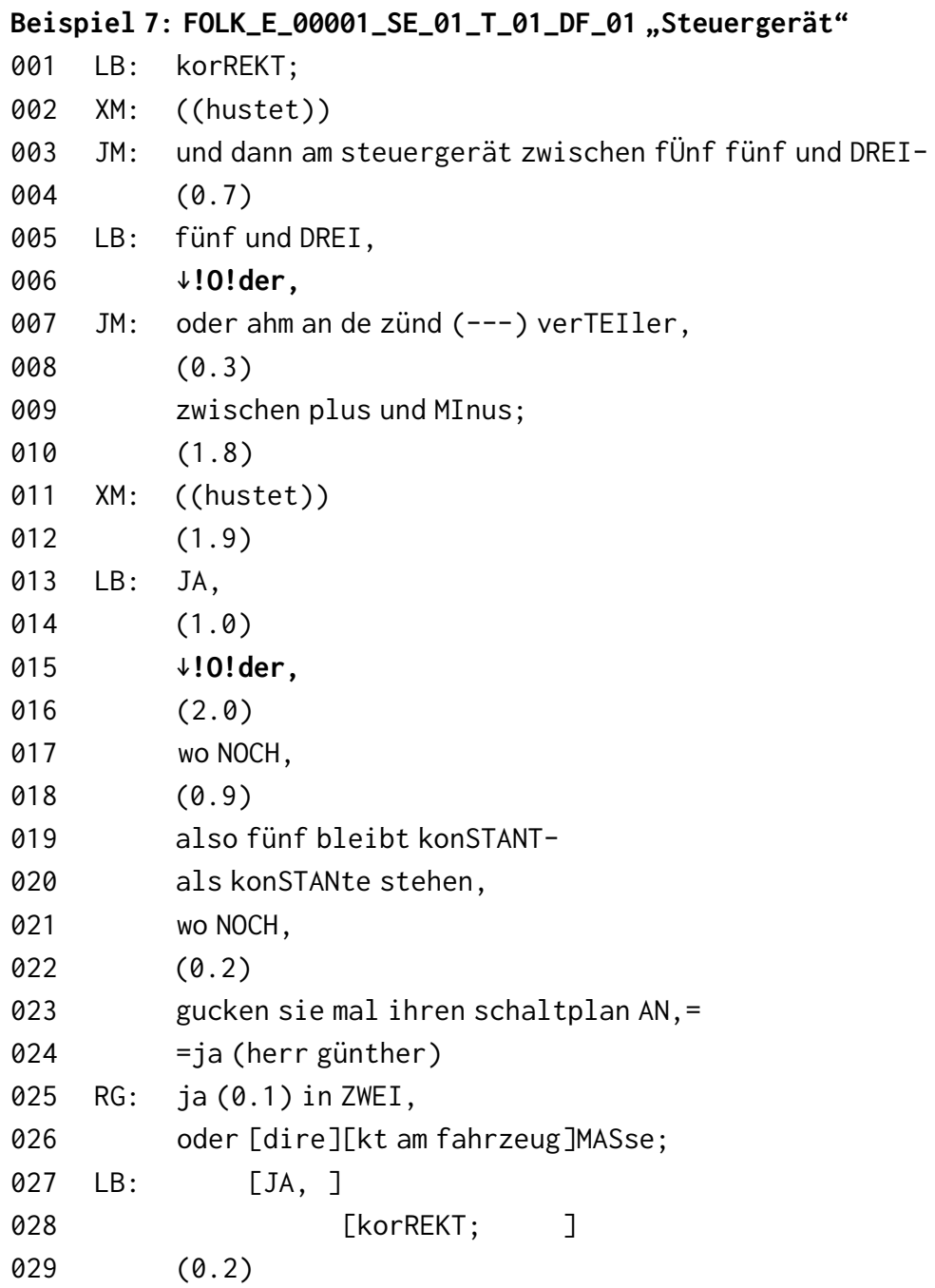


Der Lehrer LB wiederholt die Antwort des Schülers JM zunächst (005, hier als Fragment realisiert) und schließt ein !O!der, an, das in seiner prosodischen Gestaltung von den bisher betrachteten Vorkommen von finalem oder abweicht (siehe Abschnitt 3). Auch in der kontextualisierten epistemischen Haltung ist das in Segment 006 realisierte !O!der, von den bisherigen Konstruktionen mit finalem oder zu unterscheiden: Zum einen behandelt es die zuvor wiederholte Schülerantwort grundsätzlich als zutreffend. Eine alleinige Wiederholung mit final steigender Kontur hätte als Infragestellung der Richtigkeit der Schülerausführungen gehört werden können. In Kombination mit dem finalen !O!der, werden die bisherigen Ausführungen hingegen als Sicherung einer in Teilen richtigen Antwort gerahmt. Zum anderen macht !O!der, weitere Antwortalternativen relevant, die durch die SchülerInnen verbalisiert werden müssen (vgl. Koshik $2002 \mathrm{zu}$ „designedly incomplete utterances“). Die Schülerantwort war also nicht vollständig; die Existenz weiterer Antworten, die ebenfalls Gültigkeit besitzen, wird angezeigt. Während sich in den bisher betrachteten Konstruktionen mit finalem oder zumeist eine exklusive Lesart der Disjunktion rekonstruieren lässt, wird für Konstruktionen mit !O!der, also eine inklusive Lesart impliziert.

Eine solche Antwortergänzung wird im Nachgang von Schüler JM präsentiert (007). Durch ein initiales oder wird die Äußerung zum einen als kollaborative Expansion zu der vorherigen Lehreräußerung formatiert (vgl. Lerner 1991) und zum anderen explizit als Ergänzung weiterer Möglichkeiten gerahmt. Auch diese Antwort wird zunächst durch den Lehrer bestätigt (durch ein mittel steigendes „JA,“ (013), bevor dann nach kurzer Pause mit einem erneuten !O!der, (015) nach einer zusätzlichen Antworterweiterung gesucht wird. ${ }^{14}$ Als diese wiederum nicht direkt gegeben wird, fragt der Lehrer explizit nach einer zusätzlichen Stelle, an dem der Spannungswert abgelesen werden kann (,wo NOCH,“, 017). Diese Reformulierung dokumentiert, dass das zuvor platzierte!O!der eine Suche nach weiteren Möglichkeiten dargestellt hat. Die nun von dem Schüler Richard Günther $(\mathrm{RG})$ gegebene Antwort wird bestätigt und mehrfach als richtig evaluiert (027-032). An dieser Stelle wird also manifest, dass der Lehrer bereits

14 Dieses zweite !O!der, ist zum einen durch die umgebenden Pausen als selbstständige Einheit formatiert. Zum anderen wird die Proposition, zu der eine weitere Alternative präsentiert werden soll, nicht noch einmal wiederholt. Das !O!der, steht also ohne Bezugsäußerung, verweist aber auf 003 bzw. 005. 
von Beginn an über das erfragte Wissen verfügt hat und zudem die epistemische Autorität besitzt, über die Richtigkeit der Angaben zu urteilen.

Die Typizität für Unterrichts- und Prüfungsgespräche ist u. a. an dem spezifischen sequenziellen Format „Frage - Antwort - Bewertung“ erkennbar (Mehan 1979, Macbeth 2004), die auf die kommunikative Aufgabe der Wissensvermittlung zu beziehen ist: Die vollständige Antwort ist der Lehrkraft bereits bekannt. Die SchülerInnen sollen jedoch das ihnen implizit zugeschriebene Wissen oder Verständnis ausführen, um für die Lehrkraft (und die anderen SchülerInnen) Verstehen oder Wissensfortschritte zu dokumentieren. Der dritte Zug dokumentiert daher keinen Wissenszuwachs auf Seiten der Lehrkraft, sondern eine Anerkennung (oder Zurückweisung) der Antwort unter schulischen oder prüfungsbezogenen Bewer-

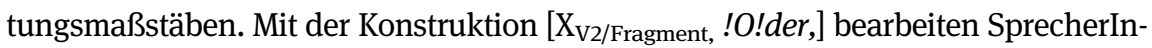
nen also die Aufgabe, vorherige Antworten auf eine ebenfalls bereits zuvor gestellte Frage aufzugreifen und in eine Wissensüberprüfung zu überführen.

\section{Diskussion}

Die Kollektionsanalyse zeigt, dass die oder-Varianten in der Kollektion rekurrent mit spezifischen Arten von Bezugsäußerungen auftreten. Dieser Befund spricht gegen eine Interpretation von finalem oder als eigenständige Diskurspartikel, die als inkrementelle Erweiterung an beliebige Äußerungen angehängt werden kann. Die enge Verzahnung der Bestandteile legt also bereits nahe, dass es sich bei den beschriebenen Gebrauchsmustern um Konstruktionen handelt.

Darüber hinaus lassen sich für die jeweiligen Kombinationen aus Bezugsäußerung und den prosodischen Varianten von oder unterschiedliche epistemische Bedeutungspotenziale beschreiben. Im Folgenden soll gezeigt werden, worin die spezifische epistemische Kontextualisierungsleistung der jeweiligen Verwendungsweisen liegt und wie diese anhand der untersuchten Gesprächsdaten bestimmt werden kann.

Folgt man bisherigen Arbeiten (Drake 2016; Willkop 1988) besteht die epistemische Haltung, die durch Äußerungen mit finalem oder ausgedrückt wird, darin, dass SprecherInnen eine relative Unsicherheit über das Zutreffen der in der Bezugsäußerung verbalisierten Proposition anzeigen. Diese epistemische Herabstufung vermittels Oder- ist für Drake $(2015,2016)$ daran ablesbar, dass Oder- eine weitere Alternative als möglich rahmt, die Präferenz für eine polare Ja/Nein-Antwort lockert und eine Antwortelaboration relevant macht. Da Äußerungen mit finalem Oder, hingegen eine polar formatierte Zustimmung präferieren, eine Alternative damit also als nicht erwartet rahmen und zudem keine extensive Elaboration relevant machen, müsste mit dieser Konstruktion eine ge- 
genüber Äußerungen mit Oder- vergleichsweise sicherere epistemische Haltung zum Ausdruck kommen.

Damit ist das Funktionspotenzial der Konstruktionen jedoch nicht hinreichend genau erfasst. Letztlich bleibt unmotiviert, warum Äußerungen mit Oder- eine weitere Alternative als möglich in Aussicht stellen, Äußerungen mit Oder, diese jedoch als unwahrscheinlicher darstellen. Allein durch die disjunktive Semantik von oder können diese Differenzen nicht erklärt werden, da diese für beide Konstruktionen anzunehmen wäre. Vielmehr zeigt sich hier, dass das Zusammenspiel zwischen den prosodischen Varianten von finalem oder und den spezifisch formatierten Bezugsäußerungen eingehender betrachtet werden muss. Evidenzen für die differenten Funktionen der oder-Konstruktionen lassen sich etwa in der Art der Eingliederung der Bezugsäußerungen in den Diskurs und der Art der verhandelten Wissensgegenstände finden. Die sequentielle Analyse der Belegstellen hat gezeigt, dass SprecherInnen sowohl bei Konstruktionen mit Oder, als auch bei Konstruktionen mit Oder-i.d. R. keine neuen Themen einführen, sondern auf vorerwähnte Gesprächsgegenstände verweisen. ${ }^{15}$ Diese Bezüge zum vorherigen Diskurs sind in den verschiedenen Konstruktionen jedoch unterschiedlich gerahmt, wie der Überblick in Tab. 2 illustriert. ${ }^{16}$

Tab. 2: Überblick Bezugsäußerungen mit oder, und oder-.

\begin{tabular}{|c|c|}
\hline Oder, & Oder- \\
\hline $\begin{array}{l}\text { hä wir ham doch AUCH luchse;=//=0der, } \\
\text { [Beispiel 1] }\end{array}$ & $\begin{array}{l}\text { und seit Elnem jahr habt habt ihr so eine so ne } \\
\text { so ne-//ja so ne kUmpel freundschaft } \\
\text { platOnisch gute beZIEhung,// } \downarrow \text { Ode:r- } \\
\text { [Beispiel 3] }\end{array}$ \\
\hline das kann doch KEIner so richtig geil; & also du hast schon verSUCHT-//ihn (0.1) äh \\
\hline$=/ /=$ Oder, [Beispiel 2] & 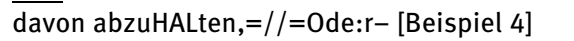 \\
\hline $\begin{array}{l}\text { dEr spielt bei LAzio;=//=0der, } \\
{[\text { KoMI \#05] }}\end{array}$ & $\begin{array}{l}\text { leben denn deine GROSSeltern noch, } \\
/ /=\langle\langle p\rangle \downarrow 0 \text { de:r } \rightarrow[\text { Beispiel } 5]\end{array}$ \\
\hline $\begin{array}{l}\text { es sind aber noch MEHR; }=/ /=0 \text { der, } \\
{[\text { [AuDa \#940] }}\end{array}$ & war das iROnisch,//=Ode:r- [Beispiel 6] \\
\hline $\begin{array}{l}\text { das sieht doch sowieSO keiner; }=/ /=0 \text { der, } \\
{[\text { KoMI \#01] }}\end{array}$ & $\begin{array}{l}\text { bist du denn STARK allergisch, //=0der- [KoMI } \\
\# 11]\end{array}$ \\
\hline aber bei dir in der Ecke-//ist doch der & und (.) da: (.) warst du dann (.) länger in der (.) \\
\hline 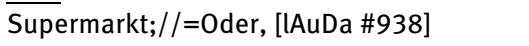 & 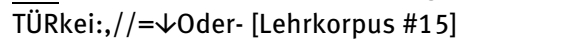 \\
\hline
\end{tabular}

15 Diese Funktion umschreibt Drake $(2016: 174,179)$ als „formulate an understanding“. Auch bei Selting (1995) lässt sich in den meisten Fällen die Tendenz zu einem thematischen Anschluss an das bisher Gesagte ausmachen.

16 Der Überblick wurde mit weiteren Belegen aus der Kollektion ergänzt. 
Der Blick auf die durch Unterstreichung hervorgehobenen Partikeln und Konnektoren lässt erkennen, dass sich Oder, auf solche Propositionen bezieht, die in der Regel nicht aus dem Gesprächsverlauf ableitbar sind und oftmals im Widerspruch zu bisherigen Sprecherannahmen stehen (u. a. markiert durch die Partikeln aber, doch). Die durch Oder- appendierten Äußerungen werden hingegen meist als themenadditiv (und) oder als Inferenz aus dem bisher Gesagten (also, denn) gerahmt. Betrachtet man die durch Fettsatz hervorgehobenen Pronomina und Possessivartikel zeigt sich ferner, dass sich Konstruktionen mit finalem Oder, und Oder- auf unterschiedliche Wissensdomänen beziehen. Während Äußerungen mit Oder, auf meist vorerwähnte Gegenstände verweisen, die interpersonell erfassbar sind (Informationen über andere Personen, Umstände etc.), werden bei Äußerungen mit Oder- Wissensbestände tangiert, die oftmals ausschließlich im Wissens- und Wahrnehmungsbereich der angesprochenen Person liegen. Dies umfasst etwa das Wissen über die eigene Biographie (Beispiele 3, 4, Lehrkorpus \#15, siehe Tab. 2), die eigene Familie (Beispiel 5), weitere persönliche Informationen (KoMI \#11, siehe Tab. 2) oder auch die intendierte Rahmung vergangener Äußerungen (Beispiel 6). Die Verankerung im Hörerwissen spiegelt sich in der Kollektion zudem darin, dass in 43 aller 58 mit Oder- appendierten Bezugsäußerungen eine direkte Adressierung des Gegenübers erfolgt. ${ }^{17}$

In Konstruktionen mit finalem Oder, und Oder- werden somit nicht ausschließlich unterschiedliche Grade an Sicherheit über die in der Bezugsäußerung ausgedrückten Propositionen kontextualisiert. Vielmehr werden darüber hinaus auch die Quelle des Wissens („epistemic access“ siehe Raymond/Heritage 2006, Stivers et al. 2011) oder die epistemische Autorität für die in der Bezugsäußerung ausgedrückten Proposition enkodiert („epistemic authority“ Raymond/Heritage 2006, „epistemic primacy“, Stivers et al. 2011). Im Fall von Konstruktionen mit finalem Oder, wird ausgedrückt, dass SprecherInnen bereits über Vorwissen bzw. Annahmen über den Gegenstand verfügen, die sie außerhalb der aktuellen Gesprächssituation erlangt haben. Dies deckt sich mit dem Befund, dass Oder, im Kontext von Verbzweitsätzen steht, die oftmals weitere Marker relativer epistemischer Sicherheit aufweisen (final fallende Tonhöhenbewegung, Partikeln wie aber, ja oder doch). Dagegen verweisen SprecherInnen in Konstruktionen mit Oder- auf Gegenstände, zu denen die SprecherInnen im Sinne einer „epistemic authority“ keinen oder einen nur eingeschränkten, nicht primären Zugang haben

17 Dem gegenüber sind nur 9 von 41 Vorkommen von Äußerungen mit appendiertem oder, direkt an das Gegenüber adressiert. Auch bei diesen werden Inhalte angesprochen, über die die SprecherInnen ein gemeinsames Vorwissen haben (etwa die Nachfrage im Gespräch zweier Freundinnen, die sich schon häufiger gegenseitig besucht haben, vgl. Beispiel lAuDa \#938 in Tab. 2). 
dürfen (biographisches Wissen etc., siehe oben). Entsprechend kann Oder- nicht nur mit deklarativen Verbzweitsätzen, sondern auch mit Verberstfragen mit final steigender Tonhöhenbewegung verwendet werden, die eine größere Unsicherheit zum Ausdruck bringen. Konstruktionen mit finalem Oder- kontextualisieren also nicht nur Unsicherheit über das Zutreffen der Bezugsäußerung, sondern zeigen zudem an, dass SprecherInnen selbst kein „Recht“ auf das verhandelte Wissen haben.

Neuere konversationsanalytische Arbeiten diskutieren ähnliche Kontextualisierungsleistungen sequenzieller Muster im Rahmen eines Modells von interaktionaler Evidenzialität (Clift 2006; Sidnell 2012). Evidenzialität ist demnach nicht allein in grammatikalisierten Affixen enkodiert (Evidenzialität als grammatische Kategorie, Aikhenvald 2003), sondern auch sequenzielle Muster können eine evidenzielle Lesart kontextualisieren (Evidenzialität als semantisch-funktionale Kategorie, Clift 2006, siehe auch Diewald/Smirnova 2010). Eine solche Analyse setzt einen weiten Begriff von Evidenzialität voraus, der sowohl Angaben zu der Verortung des Wissens (Wer hat das Wissen? Wer darf das Wissen haben?) als auch zum Ursprung des Wissens (Habe ich das Wissen erschlossen oder unabhängig von der aktuellen Situation erlangt?) sowie zu der Wissensquelle (z. B. Habe ich Wissen durch Anschauung oder durch Hörensagen?) umfasst (siehe Chafe 1986). In einem solcherart weiten und funktionalen Verständnis können demnach auch die teilschematischen Konstruktionen mit finalem oder (deren Beschreibung um Angaben zu typischen sequenziellen Kontexten angereichert ist) evidenzielle Rahmungen ausdrücken.

Aus dieser differenten Verortung des Wissens (außerhalb oder innerhalb der Sprechsituation) oder von Zugangsrechten (ausschließlich bei den HörerInnen oder bei HörerInnen und SprecherInnen) kann schließlich der Unterschied in der Sprechersicherheit zwischen Konstruktionen mit Oder, und Oder- abgeleitet werden: Wissen, das unabhängig von der Sprechsituation gewonnen wurde, kann sicherer sein als Wissen, das erst in der Sprechsituation inferiert wird und für das SprecherInnen zudem keinen primären Zugang beanspruchen dürfen. ${ }^{18}$

Anders verhält es sich hingegen für Konstruktionen mit finalem !O!der, die in den untersuchten Daten ausschließlich in Prüfungs- und Unterrichtsgesprächen belegt sind. Mit diesen Konstruktionen bringen SprecherInnen zum Ausdruck, dass sie über eine hohe epistemische Autorität verfügen. Nicht nur haben sie gesichertes Wissen über das Zutreffen der bereits durch jemand ande-

18 Hier zeigen sich die engen Bezüge, die zwischen evidenziellen und epistemischen Lesarten bestehen (vgl. Diewald/Smirnova 2010). 
ren verbalisierten Bezugsäußerung, sondern auch über die noch zu verbalisierenden Inhalte. Die Ratifikation der Antworten im dritten Turn zeugt von genau dieser epistemischen Konstellation. Den befragten SchülerInnen oder Prüflingen wird aber zugleich unterstellt, dass sie über das notwendige Wissen verfügen können, um die richtige Antwort zu geben.

Auch wenn in den Bezugsäußerungen weitere lexikalische oder grammatische Ressourcen enthalten sind, die epistemische Haltungen ausdrücken, lassen sich die verschiedenen Lesarten nicht in allen Fällen ausschließlich kompositionell aus der Formatierung der Bezugsäußerungen und des finalen oder herleiten. Nicht immer werden SprecherInnen von Äußerungen mit finalem Oder-direkt adressiert (Beispiel 6), auch bei Konstruktionen mit finalem Oder, können SprecherInnen direkt angesprochen werden (Beispiel 1AuDa \#938, siehe Tab. 2), nicht immer kontextualisieren Modalpartikeln bei Äußerungen mit finalem Oder, Widersprüche $\mathrm{zu}$ vorher als gültig angenommenem Wissen (KoMI \#05, siehe Tab. 2). Bei Belegen, die keine lexikalischen oder syntaktischen Marker enthalten, kommt der Prosodie der Bezugsäußerung und des appendierten oder eine entscheidende Rolle für die Differenzierung der verschiedenen Konstruktionen zu. Die präsentierten Befunde weisen also darauf hin, dass die jeweilige prosodische Formatierung als Merkmal der teilspezifischen Konstruktionen mit finalem oder ebenfalls zu erfassen ist (vgl. Tab. 3).

Durch ihre Funktion, verschiedene epistemische Haltungen (epistemische Autorität, Zugang zum Wissen, Sprechersicherheit) auszudrücken, sind Konstruktionen mit finalem oder auf die Lösung unterschiedlicher kommunikativer Aufgaben zugeschnitten (Günthner 2006; Imo 2010). Dies erklärt, dass Konstruktionen mit Oder- häufig in institutionellen Gesprächsgattungen wie qualitativen Interviews oder Radio-Phone-Ins vorkommen, in denen sich die am Gespräch beteiligten Personen nicht oder nicht gut kennen. In einer solchen Teilnehmerkonstellation erweisen sich Konstruktionen mit Oder- als funktional, um eine elaborierte Antwort über biographische Wissensbestände der GesprächspartnerInnen relevant zu machen. Sie fungieren als erzählgenerierende Technik, mit der die InterviewerInnen eine an vorher Gesagtes anknüpfende, weiterführende Frage stellen, zu deren Gegenstand sie über keinerlei Wissen verfügen und für deren Beantwortung sie längere Ausführungen über biographische Wissensbestände relevant machen (König i. E.).

Durch Konstruktionen mit finalem Oder, werden hingegen Annahmen oder Überzeugungen präsentiert, die außerhalb des bisherigen Gesprächs erlangt wurden, um sie einer gemeinsamen Überprüfung und ggf. Sicherung zu unterziehen. In diesem Format zeigt sich eine Aktivitätsspezifik darin, dass oftmals Widersprüche zwischen den vorherigen Gesprächsbeiträgen zugrundeliegenden Präsuppositionen und bisherigen Annahmen oder Erwartungen der Spre- 


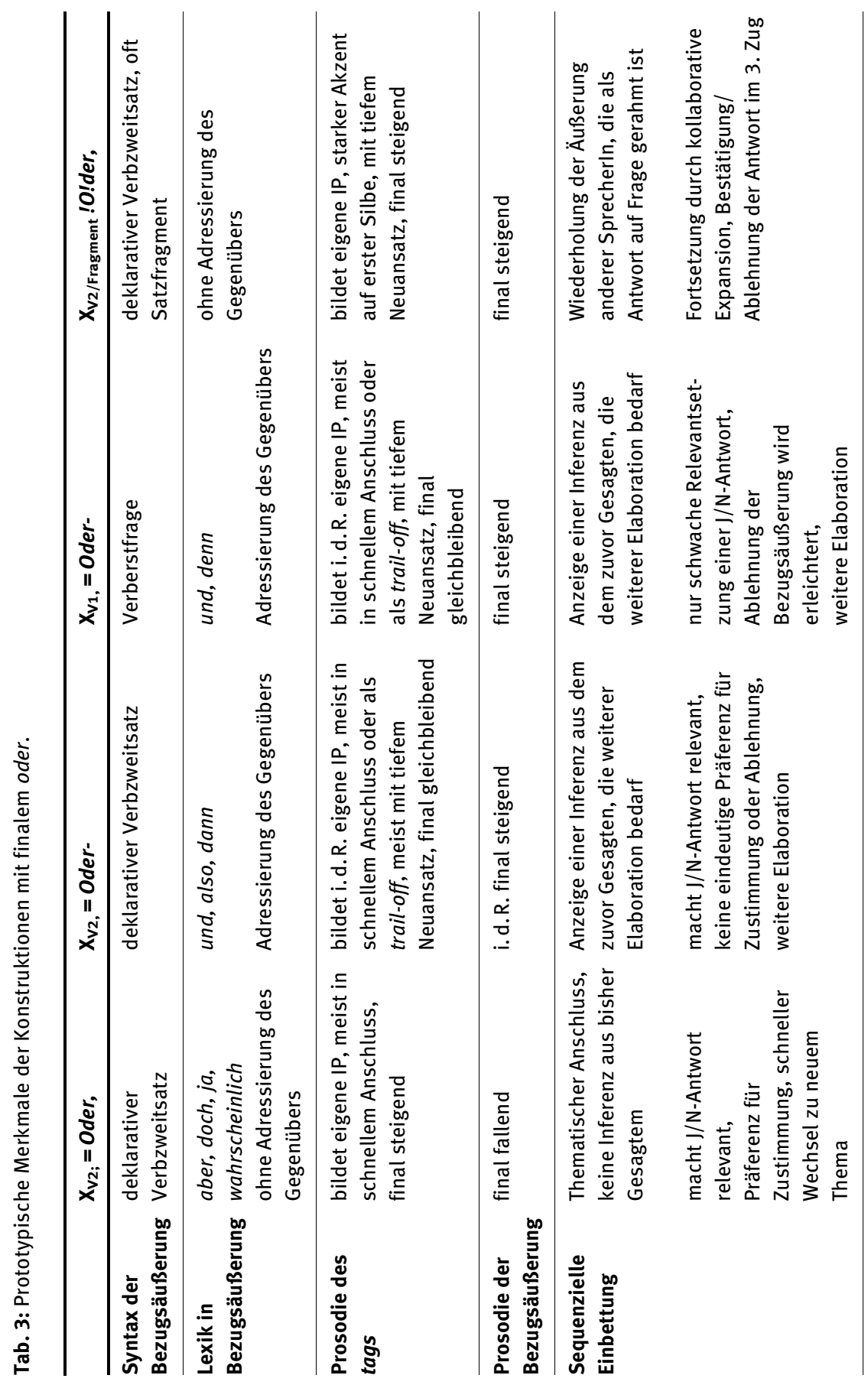




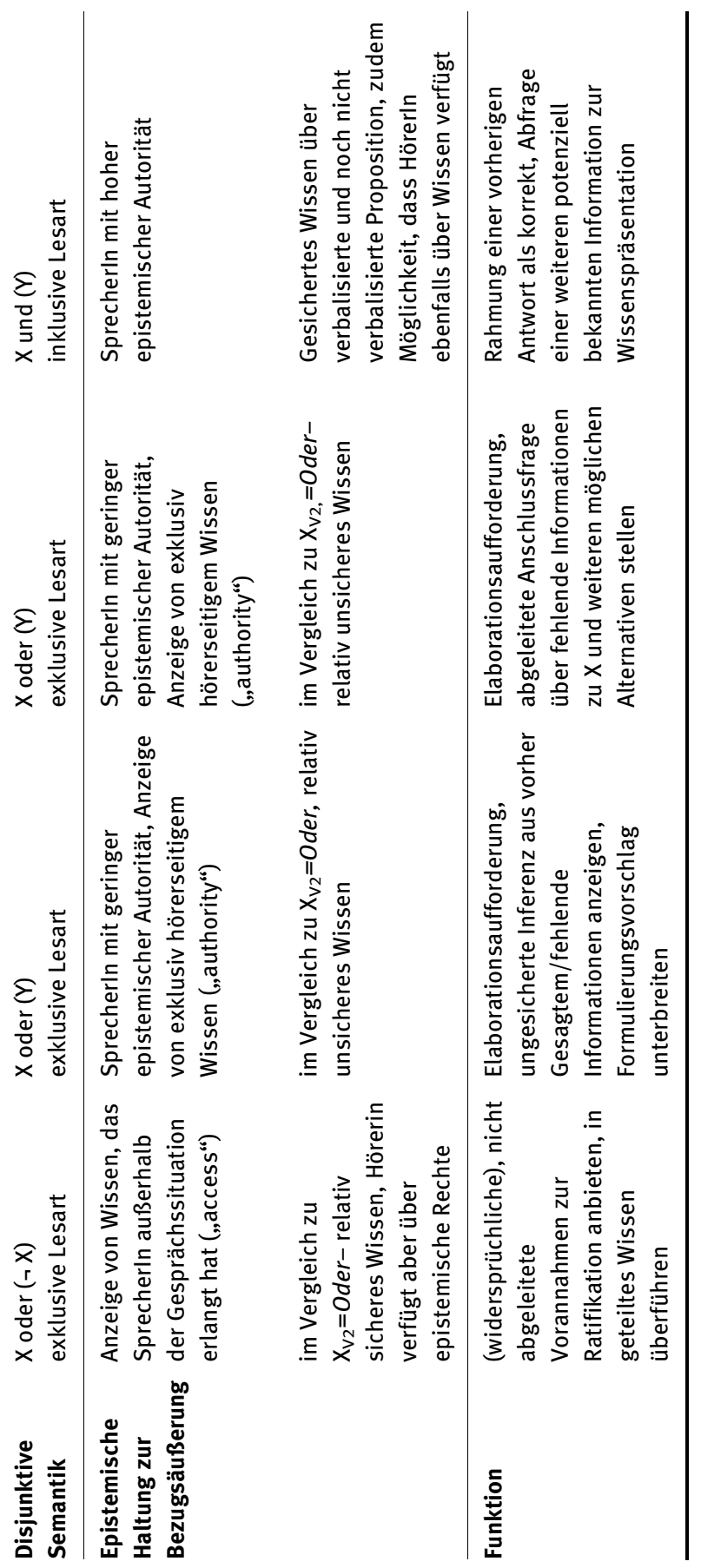


cherInnen bearbeitet werden, um zu einem geteilten Verständnis über den Gesprächsgegenstand zu gelangen.

Darüber hinaus hat die Erweiterung des Korpus um Diskurstypen wie Prüfungs- und Unterrichtsinteraktionen aufgezeigt, dass es weitere Konstruktionen mit finalem oder gibt, die sich in ihrer sequenziellen Einbettung und der prosodischen Formatierung grundlegend unterscheiden. Antworten auf Wissensfragen werden wiederholt oder rephrasiert; ein angehängtes !O!der, kontextualisiert, dass es weitere mögliche Antworten gibt, in Bezug auf die die Lehrperson über gesichertes Wissen verfügt. SchülerInnen und PrüfungskandidatInnen werden vermittels dieser Konstruktion dazu aufgefordert, ihr Wissen zu verbalisieren und zur Begutachtung zu stellen. Konstruktionen mit finalem !O!der sind also spezifisch auf die kommunikative Aufgabe der Wissensüberprüfung zugeschnitten.

Bei dem Zusammenspiel von Bezugsäußerung und finalem oder lassen sich verschiedene rekurrente Merkmale auf prosodischer, lexikalischer, syntaktischer und sequenzieller Ebene aufzeigen (vgl. Tab. 3), die auf die Verfestigung teilschematischer Konstruktionen hindeuten. Die Erfassung der Gebrauchsmuster als Konstruktionen wird zusätzlich dadurch gestützt, dass sich die verschiedenen Funktionspotenziale auch dann rekonstruieren lassen, wenn keine oder nur wenige Formmerkmale in der Bezugsäußerung realisiert sind. Die Konstruktionsbedeutung lässt sich also nicht immer vollumfänglich aus einzelnen Äußerungsbestandteilen herleiten. Der prosodischen Gestaltung kommt hingegen eine entscheidende Rolle zu. Sie ist nicht durch den jeweiligen sequenziellen Kontext motiviert, sondern ist ein zentrales Merkmal für die Unterscheidung der verschiedenen Konstruktionen mit finalem oder.

\section{Ausblick}

Während es etwa für das Englische zahlreiche Arbeiten zu finalen Konjunktionen wie but, though, or oder and gibt (vgl. Barth-Weingarten 2014; Barth-Weingarten/Couper-Kuhlen 2002; Mulder/Thompson 2008; Walker 2012), steht die interaktionale Analyse von finalen Konjunktionen im gesprochenen Deutsch erst an ihrem Anfang (vgl. Haselow 2015). Die hier untersuchten Konstruktionen mit finalem oder sind in ein Netzwerk von weiteren Konstruktionen mit question tags und finalen Markern eingebettet (König 2017), bei dem sich im Gebrauch distinkte, aber teilverwandte Form-Funktions-Profile entwickelt haben. Darüber hinaus ähnelt finales Oder- in seiner nicht eingelösten Projektion weiterer Inhalte finalen Konnektoren wie insofern (Imo 2011a), von daher (Bücker 2014) oder deswegen (König 2012). Anders als diese bringt finales oder aber eine 
epistemische Modalisierung der Bezugsäußerung zum Ausdruck und führt hierdurch in der Regel einen Sprecherwechsel herbei. Zukünftige Untersuchungen sollten also die gesamte Konstruktionsfamilie der finalen Konnektoren erfassen, um die spezifischen Leistungen der einzelnen Konstruktionen im Abgleich untereinander konturieren zu können. Ebenso sollten im Rahmen einer solchen Netzwerkanalyse weitere Konstruktionen erschlossen werden, für die sich ähnliche Antwortpräferenzen beobachten lassen. So weisen etwa die Arbeiten von Selting (1995) und Steensig/Heinemann (2013) darauf hin, dass es weitere Frageformate gibt, die zusätzlich zu einer Zustimmung eine Elaboration des Antwortturns einfordern (,yes/no+questions“, Steensig/Heinemann 2013).

Neben der Analyse einer möglichen arealen Ausdifferenzierung verschiedener Konstruktionen mit question tags (vgl. Lanwer i. V.) müssen zudem weitere Interaktionstypen untersucht werden (insbesondere im Feld der zielgeleiteten und wissenszentrierten institutionellen Kommunikation), in denen finale Konjunktionen für die Lösung jeweils spezifischer Aufgaben genutzt werden können. Es besteht die Möglichkeit, dass weitere Form-Funktions-Paare mit finalem oder aufzufinden sind, die in der vorliegenden Kollektion nicht belegt sind. Darüber hinaus kann eine multimodale Interaktionsanalyse aufzeigen, inwiefern finale Konjunktionen etwa mit Blickverhalten koordiniert werden, um in MehrparteienInteraktionen nächste SprecherInnen zu selegieren (Weiß 2018).

Arbeiten zu finalem or im Englischen (Drake 2015), finalem eller im Schwedischen (Lindström 1999) oder finalem ou Französischen (Persson 2017) dokumentieren ähnliche Konstruktionen mit disjunktiven Konjunktionen in anderen Sprachen. Eine sprachvergleichende Analyse sollte daher der Frage nachgehen, inwiefern sich in solchen Konstruktionen generische Lösungen $\mathrm{zu}$ ähnlichen kommunikativen Problemen ausgebildet haben oder inwiefern sich sprachspezifische formale und funktionale Verfestigungen beschreiben lassen. Hierfür kann es relevant sein, mögliche Entstehungskontexte für die verschiedenen Gebrauchsmuster zu rekonstruieren: Bislang wurden ausschließlich solche Vorkommen von finalem oder untersucht, auf die ein Sprecherwechsel erfolgt. Damit lässt man jedoch solche Kontexte außer Acht, in denen eine mit oder begonnene TCU inkrementell komplettiert wird. In solchen Turn-Fortführungen könnte ein Kontext gegeben sein, aus dem heraus sich oder von einem Haltesignal zur Anzeige einer Wortsuche zu einer finalen Partikel zur Anzeige verschiedener epistemisch-evidenzieller Haltungen grammatikalisiert hat. 


\section{Literatur}

Aikhenvald, Alexandra Y. (2003): Evidentiality in typological perspective. In: Aikhenvald, Alexandra Y. und R. M. W. Dixon (Hrsg.): Studies in Evidentiality. Amsterdam: Benjamins, $1-31$.

Auer, Peter (2016): „Wie geil ist das denn?“. Eine neue Konstruktion im Netzwerk ihrer Nachbarn. In: Zeitschrift für germanistische Linguistik 44(1), 69-92.

Barth-Weingarten, Dagmar (2011): Response tokens in interaction - prosody, phonetics and a visual aspect of German JAJA. In: Gesprächsforschung. Online-Zeitschrift zur verbalen Interaktion 12, 301-370.

Barth-Weingarten, Dagmar (2014): Dialogism and the emergence of final particles: The case of and. In: Günthner, Susanne, Wolfgang Imo und Jörg Bücker (Hrsg.): Grammar and Dialogism. Sequential, Syntactic, and Prosodic Patterns between Emergence and Sedimentation. Berlin/Boston: de Gruyter, 335-366.

Barth-Weingarten, Dagmar (2016): Intonation Units Revisited. Cesuras in talk-in-interaction. Amsterdam: Benjamins.

Barth-Weingarten, Dagmar und Elizabeth Couper-Kuhlen (2002): On the development of final though. A case of grammaticalization? In: Wischer, Ilse und Gabriele Diewald (Hrsg.): New Refelctions on Grammaticalization. Amsterdam: Benjamins, 345-361.

Betz, Emma und Arnulf Deppermann (2018): Indexing Priority of Position: Eben as Response Particle in German. In: Research on Language \& Social Interaction 51(2), 171-193.

Bolden, Galina B. (2010): 'Articulating the unsaid' via and-prefaced formulations of others' talk. In: Discourse Studies 12(1), 5-32.

Bücker, Jörg (2014): Konstruktionen und Konstruktionscluster: das Beispiel der Zirkumposition von XP her im gesprochenen Deutsch. In: Lasch, Alexander und Alexander Ziem (Hrsg.): Grammatik als Netzwerk von Konstruktionen. Sprachwissen im Fokus der Konstruktionsgrammatik. Berlin: de Gruyter, 117-136.

Bücker, Jörg, Susanne Günthner und Wolfgang Imo (Hrsg.) (2015): Konstruktionsgrammatik V: Konstruktionen im Spannungsfeld von sequenziellen Mustern, kommunikativen Gattungen und Textsorten. Tübingen: Stauffenburg.

Chafe, Wallace (1986): Evidentiality in English conversation and academic writing. In: Chafe, Wallace und Johanna Nichols (Hrsg.): Evidentiality: The Linguistic Coding of Epistemology. Norwood: Ablex, 261-272.

Clift, Rebecca (2006): Indexing stance. Reported speech as an interactional evidential. In: Journal of Sociolinguistics 10(5), 569-595.

Couper-Kuhlen, Elizabeth (2012): Some truths and untruths about final intonation in conversational questions. In: Ruiter, Jan P. de (Hrsg.): Questions. Formal, functional and interactional perspectives. Cambridge: Cambridge University Press, 123-145.

Deppermann, Arnulf (2009): Verstehensdefizit als Antwortverpflichtung: Interaktionale Eigenschaften der Modalpartikel denn in Fragen. In: Günthner, Susanne und Jörg Bücker (Hrsg.): Grammatik im Gespräch. Konstruktionen der Selbst- und Fremdpositionierung. Berlin: de Gruyter, 23-56.

Deppermann, Arnulf (2011): Konstruktionsgrammatik und Interaktionale Linguistik: Affinitäten, Komplementaritäten und Diskrepanzen. In: Lasch, Alexander und Alexander Ziem (Hrsg.): Konstruktionsgrammatik III. Aktuelle Fragen und Lösungsansätze. Tübingen: Stauffenburg, 207-240. 
Deppermann, Arnulf (2015): Wissen im Gespräch: Voraussetzung und Produkt, Gegenstand und Ressource. In: InLiSt 57, 1-31.

Deppermann, Arnulf und Henrike Helmer (2013): Zur Grammatik des Verstehens im Gespräch. Inferenzen anzeigen und Handlungskonsequenzen ziehen mit also und dann. In: Zeitschrift für Sprachwissenschaft 32 (1), 1-39.

Diewald, Gabriele und Kerstin Fischer (1998): Zur diskursiven und modalen Funktion der Partikeln aber, auch, doch und $j a$ in Instruktionsdialogen. In: Linguistica 38(1), 75-99.

Diewald, Gabriele und Elena Smirnova (2010): Evidentiality in German. Linguistic Realization and Regularities in Grammaticalization. Berlin: de Gruyter Mouton.

Drake, Veronika (2015): Indexing uncertainty. The case of turn-final or. In: Research on Language and Social Interaction 48(3), 301-318.

Drake, Veronika (2016): German questions and turn-final oder. In: Gesprächsforschung. Online-Zeitschrift zur verbalen Interaktion 17, 168-195.

Duden (2016): Die Grammatik. Unentbehrlich für richtiges Deutsch. Berlin: Dudenverlag.

Fillmore, Charles J., Paul Kay, Paul und Mary Catherine O'Connor (1988): Regularity and idiomaticity in grammatical constructions. The case of Let Alone. In: Language 64(3), 501-538.

Glaser, Elvira (2003): „Is de für Herrn König, oder?“. Zur Sprachgeographie von Gliederungspartikeln. In: Funk, Edith et al. (Hrsg.): Sprachgeschichten. Ein Lesebuch für Werner König zum 60. Geburtstag. Heidelberg: Winter, 63-79.

Goldberg, Adele E. (1995): Constructions. A Construction Grammar Approach to Argument Structure. Chicago: University of Chicago Press.

Günthner, Susanne (2006): Von Konstruktionen zu kommunikativen Gattungen. Die Relevanz sedimentierter Muster für die Ausführung kommunikativer Aufgaben. In: Deutsche Sprache 34(1-2), 173-190.

Günthner, Susanne (2009): Konstruktionen in der kommunikativen Praxis - Zur Notwendigkeit einer interaktionalen Anreicherung konstruktionsgrammatischer Ansätze. In: Zeitschrift für germanistische Linguistik 37(3), 402-426.

Günthner, Susanne (2017): Diskursmarker in der Interaktion - Formen und Funktionen univerbierter guck mal- und weißt du-Konstruktionen. In: Blühdorn, Hardarik et al. (Hrsg.): Diskursmarker im Deutschen. Reflexionen und Analysen. Göttingen: Verlag für Gesprächsforschung, 103-130.

Hagemann, Jörg (2009): Tag questions als Evidenzmarker. Formulierungsdynamik, sequentielle Struktur und Funktionen redezuginterner tags. In: Gesprächsforschung. Online-Zeitschrift zur verbalen Interaktion 10, 145-176.

Haselow, Alexander (2015): Final particles in spoken German. In: Hancil, Sylvie, Alexander Haselow und Margje Post (Hrsg.): Final particles. Berlin: de Gruyter Mouton, 77-107.

Helbig, Gerhard und Joachim Buscha (2013): Deutsche Grammatik. Ein Handbuch für den Ausländerunterricht. Berlin/München: Langenscheidt.

Heritage, John (2013): Epistemics in conversation. In: Sidnell, Jack und Tanya Stivers (Hrsg.): The Handbook of Conversation Analysis. Chichester: Blackwell, 370-394.

Hoffmann, Ludger (2013): Deutsche Grammatik. Grundlagen für Lehrerausbildung, Schule, Deutsch als Zweitsprache und Deutsch als Fremdsprache. Berlin: Erich Schmidt Verlag.

Imo, Wolfgang (2009): Konstruktion oder Funktion? Erkenntnisprozessmarker (change-ofstate-token) im Deutschen. In: Günthner, Susanne und Jörg Bücker (Hrsg.): Grammatik im Gespräch. Konstruktionen der Selbst- und Fremdpositionierung. Berlin: de Gruyter: 57-86. 
Imo, Wolfgang (2010): „Mein Problem ist/mein Thema ist” ('My problem is/my topic is'): How syntactic patterns and genres interact. In: Dorgeloh, Heidrun und Anja Wanner (Hrsg.): Syntactic Variation and Genre. Berlin/New York: de Gruyter Mouton, 141-166.

Imo, Wolfgang (2011a): Cognitions are not observable but their consequences are: Mögliche Aposiopese-Konstruktionen in der gesprochenen Altagssprache. In: Gesprächsforschung. Online-Zeitschrift zur verbalen Interaktion 12, 265-300.

Imo, Wolfgang (2011b): „Jetzt gehen wir einen trinken, gell?“ Vergewisserungssignale (tag questions) und ihre Relevanz für den DaF-Unterricht. In: Moraldo, Sandro M. (Hrsg.): Deutsch aktuell 2. Einführung in die Tendenzen der deutschen Gegenwartssprache. Rom: Carocci, 127-150.

Imo, Wolfgang (2012): Wortart Diskursmarker? In: Rothstein, Björn (Hrsg.): Nicht-flektierende Wortarten. Berlin/Boston: de Gruyter, 48-88.

Imo, Wolfgang (2016): Satzmodus, Konstruktion oder keines von beidem? Äußerungsformen und Äußerungsbedeutungen in interaktionaler gesprochener Sprache. In: Finkbeiner, Rita und Jörg Meibauer (Hrsg.): Satztypen und Konstruktionen. Berlin/Boston: de Gruyter, 373-405.

Jefferson, Gail (1981): The abominable Ne? An exploration of post-response pursuit of response. In: Schröder, Peter und Hugo Steger (Hrsg.): Dialogforschung. Düsseldorf: Pädagogischer Verlag Schwann, 53-88.

König, Katharina (i. E.): Die gemeinsame Konstruktion von dialektbezogenem Wissen und Sprachbewertungen in den Interviews aus dem SiN-Korpus. In: Denkler, Markus und Jens Lanwer (Hrsg.): Dialektologie und Gesprächslinguistik. Hildesheim: Olms.

König, Katharina (2012): Formen und Funktionen von syntaktisch desintegriertem deswegen im gesprochenen Deutsch. In: Gesprächsforschung. Online-Zeitschrift zur verbalen Interaktion 13, 45-71.

König, Katharina (2017): Question tags als Diskursmarker? - Ansätze zu einer systematischen Beschreibung von ne im gesprochenen Deutsch. In: Blühdorn, Hardarik et al. (Hrsg.): Diskursmarker im Deutschen. Reflexionen und Analysen. Göttingen: Verlag für Gesprächsforschung, 233-258.

Koshik, Irene (2002): Designedly incomplete utterances: A pedagogical practice for eliciting knowledge displays in error correction sequences. In: Research on Language and Social Interaction 35(3), 277-309.

Lanwer, Jens (i. V.): Alignmentmarker in nord(west)deutscher Alltagssprache. In: Niederdeutsches Wort 59.

Lerner, Gene H. (1991): On the syntax of sentences-in-progress. In: Language in Society 20, 441-458.

Lindström, Anna (1999): Language as social action. Grammar, prosody, and interaction in Swedish conversation. Uppsala: Uppsala University.

Linell, Per (2009): Grammatical constructions in dialogue. In: Bergs, Alexander und Gabriele Diewald (Hrsg.): Contexts and Constructions. Amsterdam: Benjamins, 97-110.

Macbeth, Douglas (2004): The relevance of repair for classroom correction. In: Language in Society 33(5), 703-736.

Mehan, Hugh (1979): “What time is it, Denise?" Asking known information questions in classroom discourse. In: Theory Into Practice 18(4), 285-294.

Mulder, Jean und Sandra A. Thompson (2008): The grammaticization of but as a final particle in English conversation. In: Laury, Ritva (Hrsg.): Crosslinguistic Studies of Clause Combining. Amsterdam: Benjamins, 179-204. 
Ogden, Richard (2010): Prosodic constructions in making complaints. In: Barth-Weingarten, Dagmar, Elisabeth Reber und Margret Selting (Hrsg.): Prosody in Interaction. Amsterdam: Benjamins, 81-103.

Oloff, Florence (2017): Genau als redebeitragsinterne, responsive, sequenzschließende oder sequenzstrukturierende Bestätigungspartikel im Gespräch. In: Blühdorn, Hardarik et al. (Hrsg.): Diskursmarker im Deutschen. Reflexionen und Analysen. Göttingen: Verlag für Gesprächsforschung, 207-232.

Persson, Rasmus (2017): Tentatively designed polar questions and their responses. Vortrag bei der 15th International Pragmatics Association Conference, Belfast.

Raymond, Geoffrey und John Heritage (2006): The epistemics of social relations. Owning grandchildren. In: Language in Society 35(5), 677-705.

Selting, Margret (1995): Prosodie im Gespräch. Aspekte einer interaktionalen Phonologie der Konversation. Tübingen: Niemeyer.

Selting, Margret (2004): Listen: Sequenzielle und prosodische Struktur einer kommunikativen Praktik - eine Untersuchung im Rahmen der Interaktionalen Linguistik. In: Zeitschrift für Sprachwissenschaft 23, 1-46.

Selting, Margret et al. (2009): Gesprächsanalytisches Transkriptionssystem 2 (GAT 2). In: Gesprächsforschung. Online-Zeitschrift zur verbalen Interaktion 10, 353-402.

Sidnell, Jack (2012): Who knows best. Evidentiality and epistemic asymmetry in conversation. In: Pragmatics and Society 3(2), 294-320.

Steensig, Jakob und Trine Heinemann (2013): When 'yes' is not enough - as an answer to a yes/ no question. In: Szczepek Reed, Beatrice und Geoffrey Raymond (Hrsg.): Units of Talk Units of Action. Amsterdam: Benjamins, 207-242.

Stivers, Tanya, Lorenza Mondada und Jakob Steensig (2011): Knowledge, morality and affiliation in social interaction. In: Stivers, Tanya, Lorenza Mondada und Jakob Steensig (Hrsg.): The Morality of Knowledge in Conversation. Cambridge: Cambridge University Press, 3-24.

Walker, Gareth (2012): Coordination and interpretation of vocal and visible resources: 'trailoff' conjunctions. In: Language and speech 55(1), 141-163.

Weinrich, Harald (2007): Textgrammatik der deutschen Sprache. Hildesheim: Georg Olms Verlag.

Weiß, Clarissa (2018): When gaze-selected next speakers do not take the turn. In: Journal of Pragmatics 133, 28-44.

Willkop, Eva-Maria (1988): Gliederungspartikeln im Dialog. München: Iudicium.

Zifonun, Gisela et al. (1997): Grammatik der deutschen Sprache. Berlin/New York: de Gruyter. 
